\title{
LIE SUPERGROUP ACTIONS ON SUPERMANIFOLDS
}

\author{
CHARLES P. BOYER AND O. A. SÁNCHEZ VALENZUELA
}

\begin{abstract}
Lie supergroups are here understood as group objects in the category of supermanifolds (as in [2, 5, and 15]). Actions of Lie supergroups in supermanifolds are defined by means of diagrams of supermanifold morphisms. Examples of such actions are given. Among them emerge the linear actions discussed in [2, 5, and 12] and the natural actions on the Grassmannian supermanifolds studied in [6-9 and 13]. The nature of the isotropy subsupergroup associated to an action is fully elucidated; it is exhibited as an embedded subsupergroup in the spirit of the theory of smooth manifolds and Lie groups and with no need for the Lie-Hopf algebraic approach of Kostant in [3]. The notion of orbit is also discussed. Explicit calculations of isotropy subsupergroups are included. Also, an alternative proof of the fact that the structural sheaf of a Lie supergroup is isomorphic to the sheaf of sections of a trivial exterior algebra bundle is given, based on the triviality of its supertangent bundle.
\end{abstract}

\section{INTRODUCTION}

The first treatise on Lie supergroups and superhomogeneous spaces was given by Kostant in his pioneering work [3]. His approach is based on exploiting the natural Hopf-superalgebra structure of the universal enveloping algebra of a given Lie superalgebra. While very efficient in proving most of the hard theorems of Lie theory in the supermanifold setting, Kostant's methods are somewhat cumbersome in dealing with specific examples. In fact, the examples that have appeared in the literature [6-9 and 13] have been treated along the lines of Berezin, Leites, and Manin [2, 4-9] rather than those of Kostant. We found, however, that there is still no good differential geometric treatment of Lie supergroup actions that parallels Kostant's Lie-Hopf theoretic approach.

In this paper we approach Lie supergroups and their actions on supermanifolds in the spirit of differential geometry; we have been guided by Kostant's results but have followed the methods of Berezin, Leites, and Manin (cf. [2, 4, 5]; see also [15]). Our original intent was to develop a theory of connections in principal bundles in the supermanifold setting and eventually give a geometric description of the moduli of superinstantons that might complement the cohomological picture already developed by Manin (see [6-9]). We were thus led to the problem of describing first the isotropy subsupergroup of an action, say

Received by the editors October 5, 1988 and, in revised form, January 1, 1989.

1980 Mathematics Subject Classification (1985 Revision). Primary 58A50.

Supported in part by research grants: NSF DMS-8815581 and CONACYT A128CC0E890045 (MT-2). 
$\psi:\left(G, A_{G}\right) \times\left(M, A_{M}\right) \rightarrow\left(M, A_{M}\right)$. Our solution goes as follows: for each $p \in M$, we make sense of the partial map, $\psi_{p}:\left(G, A_{G}\right) \rightarrow\left(M, A_{M}\right)$, and of the constant map, $\mathscr{E}_{p}:\left(G, A_{G}\right) \rightarrow\left(M, A_{M}\right)$, whose image is the $(0,0)$-dimensional point $(p, \mathbf{R})$ of $\left(M, A_{M}\right)$. Then the isotropy subsupergroup at $p,\left(G_{p}, A_{G_{p}}\right)$, is obtained by making sense of the locus in $\left(G, A_{G}\right)$ where $\psi_{p}=\mathscr{E}_{p}$. It turns out to be an embedded subsupermanifold of $\left(G, A_{G}\right)$ and it inherits naturally a Lie supergroup structure. The precise statement is technically somewhat delicate (see Theorem 4.6), but we show by concrete examples that it is a straightforward task to compute the isotropy subsupergroup of a given action; in fact, our approach has been designed so as to do this just as in the smooth theory (see $\S 5)$. Then, following the ideas in [3], it is immediate to define a supermanifold sheaf, $A_{G / G_{p}}$, on the space of cosets $G / G_{p}$, thus giving rise to a superhomogeneous space. There is also a morphism $\left(G / G_{p}, A_{G / G_{p}}\right) \rightarrow\left(M, A_{M}\right)$ naturally induced by $\psi_{p}$. The orbit through $p,\left(O_{p}, A_{O_{p}}\right)$, is the image of $\psi_{p}$ in the category of supermanifolds (cf. 4.9); it is a subsupermanifold of $\left(M, A_{M}\right)$ and its supermanifold structure is the one that makes $\left(G / G_{p}, A_{G / G_{p}}\right) \rightarrow\left(O_{p}, A_{O_{p}}\right)$ into a superdiffeomorphism.

As most of the arguments involve the propagation of germs of superfunctions defined over a neighborhood of the identity to the whole group, we provide as a technical preliminary the notions of left and right translations (resp. invariance) in the form that suits our needs. In particular, we prove quite generally that the supertangent bundle (in the sense of [11]) of a Lie supergroup is trivial. The trivialization is accomplished-as expected-by the existence of a basis of left invariant superderivations (i.e., in sheaf-theoretical terms, by proving that the Lie superalgebra of a Lie supergroup is isomorphic to a constant sheaf). This is then used to pick up function and exterior factors globally [3]; hence, the structural sheaf of a Lie supergroup is exhibited from the outset in the trivial form of Batchelor [1]. The referee has brought to our attention the fact that this result has been known to the experts since 1974 and that it was first proved by J. Bernstein in the USSR. The result also appears in Kostant's paper [3] of 1975. Our approach has thus provided an alternative proof of it (see Proposition 2.9 and Corollary 2.10 below).

Acknowledgments. We are deeply grateful to Professor Thomas Schmitt for pointing out to us the equivalence between Kostant's definition of supermanifold and that of Berezin and Leites (see [2-5]). In our original manuscript we had mistakenly stated that Kostant's definition was more general. Also, one of us (O.A.S.V.) would like to thank Professor R. Berlanga for some illuminating discussions and his most valuable criticism. Finally, we would like to thank the referee, whose comments and suggestions contributed to making this work into a more concise exposition.

\section{Abstract characterization of a Lie supergroup}

We shall understand supermanifolds and morphisms between them as in [2] and [4-7] (acquaintance with references [2-7] will be assumed). A supermani- 
foldmorphism from $\left(M, A_{M}\right)$ into $\left(N, A_{N}\right)$ is a pair, $\varphi=\left(\tilde{\varphi}, \varphi^{\#}\right)$, consisting of a continuous map, $\tilde{\varphi}: M \rightarrow N$, and a sheaf homomorphism over $N$, $\varphi^{\#}: A_{N} \rightarrow \tilde{\varphi}_{*} A_{M}$, which is local on each stalk. We shall make extensive use of the fact that a supermanifold morphism is completely determined by the superalgebra morphism on global sections, $\varphi^{\#}: A_{N}(N) \rightarrow A_{M}\left(\tilde{\varphi}^{-1}(N)\right)$, that the sheaf homomorphism gives rise to (see [2-4]). If $\left(M, A_{M}\right)$ is a $C^{\infty}$ supermanifold, $\delta:\left(M, C_{M}^{\infty}\right) \rightarrow\left(M, A_{M}\right)$ will denote the morphism determined by the canonical projection $A_{M}(M) \rightarrow C_{M}^{\infty}(M), f \mapsto \delta(f)=\tilde{f}$. Each point $p \in M$ defines a morphism, $\delta_{p}:(\{*\}, \mathbf{R}) \rightarrow\left(M, A_{M}\right)$, by letting $\delta_{p}^{\#} f=\tilde{f}(p)$; every superalgebra morphism $A_{M}(M) \rightarrow \mathbf{R}$ is of this form. The object $(\{*\}), \mathbf{R})$ is the supermanifold consisting of a single point and the constant sheaf $\mathbf{R}$, the reals, over it. It is a terminal object, for there is only one constant morphism, $\left.C_{\left(M, A_{M}\right)}:\left(M, A_{M}\right) \rightarrow(\{*\}), \mathbf{R}\right)$, from any supermanifold into it, namely the one determined by the $\mathbf{R}$-superalgebra map $\mathbf{R} \rightarrow A_{M}(M)$, $C_{\left(M, A_{M}\right)^{*}} \lambda \lambda=\lambda 1_{A_{M}(M)}$.

1.1. Definition. A Lie supergroup is a finite-dimensional supermanifold $\left(G, A_{G}\right)$ equipped with the following additional structure (see $\left.[2,5,15]\right)$ :

(i) A supermanifold morphism

$$
\mu:\left(G, A_{G}\right) \times\left(G, A_{G}\right) \rightarrow\left(G, A_{G}\right)
$$

satisfying the associativity property

$$
\mu \circ\left(\pi_{1} \times \mu \circ\left(\pi_{2} \times \pi_{3}\right)\right)=\mu \circ\left(\mu \circ\left(\pi_{1} \times \pi_{2}\right) \times \pi_{3}\right) .
$$

[Both sides are morphisms $\left(G, A_{G}\right) \times\left(G, A_{G}\right) \times\left(G, A_{G}\right) \rightarrow\left(G, A_{G}\right)$; $\pi_{i}$ denotes the projection of $\left(G, A_{G}\right) \times\left(G, A_{G}\right) \times\left(G, A_{G}\right)$ onto the $i$ th factor $(i=1,2,3)$.]

(ii) A distinguished point in the underlying manifold, $e \in G$, and hence a distinguished supermanifold morphism

$$
\mathscr{E}_{e}:=\delta_{e} \circ C_{\left(G, A_{G}\right)}:\left(G, A_{G}\right) \rightarrow\left(G, A_{G}\right)
$$

that satisfies the identity property

$$
\mu \circ\left(\mathrm{id} \times \mathscr{E}_{e}\right)=\mathrm{id}=\mu \circ\left(\mathscr{E}_{e} \times \mathrm{id}\right) .
$$

(iii) An involutive superdiffeomorphism

$$
\sigma:\left(G, A_{G}\right) \rightarrow\left(G, A_{G}\right)
$$

that satisfies the inverse property

$$
\mu \circ(\mathrm{id} \times \sigma)=\mathscr{E}_{e}=\mu \circ(\sigma \times \mathrm{id}) .
$$

Among the first few and easiest examples of Lie groups one finds in the $C^{\infty}$ category are the abelian groups defined by addition of vectors in a vector space. Their natural generalization is the following: 
1.2. Example. $\mathbf{R}^{m \mid n}=\left(\mathbf{R}^{m}, R^{m \mid n}\right)$ or, following [2], $\mathfrak{G}_{n}\left(\mathbf{R}^{m}\right)$ with additive structure.

Recall that the sheaf $R^{m \mid n}$ of the superaffine space $\mathbf{R}^{m \mid n}$ is (cf. [2-5])

$$
C_{\mathbf{R}^{m}}^{\infty} \otimes \bigwedge\left[\theta_{1}, \ldots, \theta_{n}\right]
$$

where $\left\{\theta_{1}, \ldots, \theta_{n}\right\}$ is a set of generators for an $n$-dimensional vector space over $\mathbf{R}$. Thus, for any nonempty open subset $U \subset \mathbf{R}^{m}, R^{m \mid n}(U)=C_{\mathbf{R}^{m}}^{\infty}(U) \otimes$ $\bigwedge\left[\theta_{1}, \ldots, \theta_{n}\right]$.

Let $\left\{\mathbf{e}_{1}, \ldots, \mathbf{e}_{m}\right\}$ be a basis of $\mathbf{R}^{m}$ and let $\left\{x^{1}, \ldots, x^{m}\right\}$ be the dual basis. Then, $\left\{x^{1}, \ldots, x^{m} ; \theta_{1}, \ldots, \theta_{n}\right\}$ is a global coordinate system for the supermanifold $\mathbf{R}^{m \mid n}$. We shall keep this coordinate system fixed.

Associated to the vector spaces $\mathbf{R}^{m}$ and $\Lambda\left[\theta_{1}, \ldots, \theta_{n}\right]$ there is a natural morphism

$$
\mu:\left(\mathbf{R}^{m}, R^{m \mid n}\right) \times\left(\mathbf{R}^{m}, R^{m \mid n}\right) \rightarrow\left(\mathbf{R}^{m}, R^{m \mid n}\right)
$$

defined over each nonempty open subset $U \subset \mathbf{R}^{m}$ as the one that corresponds to the superalgebra morphism $\mu^{\#}: R^{m \mid n}(U) \rightarrow R^{m \mid n} \times R^{m \mid n}(U \times U)$ given in terms of the coordinates above by

$$
\begin{array}{ll}
\mu^{\#} x^{i}=p_{1}^{\#} x^{i}+p_{2}^{\#} x^{i}, & i=1, \ldots, m, \\
\mu^{\#} \theta_{\nu}=p_{1}^{\#} \theta_{\nu}+p_{2}^{\#} \theta_{\nu}, & \nu=1, \ldots, n,
\end{array}
$$

$p_{i}:\left(\mathbf{R}^{m}, R^{m \mid n}\right) \times\left(\mathbf{R}^{m}, R^{m \mid n}\right) \rightarrow\left(\mathbf{R}^{m}, R^{m \mid n}\right)$ being the projection morphism onto the $i$ th factor $(i=1,2)$.

The origin $e=(0, \ldots, 0) \in \mathbf{R}^{m}$ is obviously a distinguished point for which the morphism $\mathscr{E}_{e}$ has the identity property. Furthermore, there is an involutive isomorphism $\sigma:\left(\mathbf{R}^{m}, R^{m \mid n}\right) \rightarrow\left(\mathbf{R}^{m}, R^{m \mid n}\right)$ having the inverse property, namely the one defined over each nonempty open subset $U \subset \mathbf{R}^{m}$ via the superalgebra morphism $\sigma^{\#}: R^{m \mid n}(U) \rightarrow R^{m \mid n}(U)$, whose effect on the fixed set of coordinates is

$$
\begin{array}{ll}
\sigma^{\#} x^{i}=-x^{i}, & i=1, \ldots, m, \\
\sigma^{\#} \theta_{\nu}=-\theta_{\nu}, & \nu=1, \ldots, n .
\end{array}
$$

Particular case: $m=0$. The sheaf $R^{0 \mid n}$ becomes the constant sheaf, $\bigwedge\left[\theta_{1}, \ldots, \theta_{n}\right]$, over $\{*\}$ and there is only one choice for $\mathscr{E}_{e}, \mu$, and $\sigma$, namely those given by the expressions above in the odd coordinates $\theta_{\nu}, \nu=1, \ldots, n$. What results is then the $(0, n)$-dimensional abelian Lie supergroup.

1.3. Example. $\mathfrak{G}_{1}\left(\mathbf{R}^{*}\right)=\left(\mathbf{R}^{*},\left.R^{1 \mid 1}\right|_{\mathbf{R}^{*}}\right)$, with a multiplicative supergroup structure; here, $\mathbf{R}^{*}=\mathbf{R}-\{0\} \simeq \mathrm{GL}(1)$.

In this case, the morphism $\mu$ is the supermultiplication morphism of $\mathbf{R}^{1 \mid 1}=$ $\left(\mathbf{R}, R^{1 \mid 1}\right)$ restricted to the open subsupermanifold $\left(\mathbf{R}^{*},\left.R^{1 \mid 1}\right|_{\mathbf{R}^{*}}\right)$. In terms of 
the standard coordinate system $\{x, \theta\}$ of $\mathbf{R}^{1 / 1}$ it is defined as (see [11])

$$
\begin{aligned}
& \mu^{\#} x=p_{1}^{\#} x p_{2}^{\#} x+p_{1}^{\#} \theta p_{2}^{\#} \theta, \\
& \mu^{\#} \theta=p_{1}^{\#} x p_{2}^{\#} \theta+p_{1}^{\#} p_{2}^{\#} x .
\end{aligned}
$$

The distinguished point in $\mathbf{R}^{*}$ is the unit $1 \in \mathbf{R}^{*}$, and the superdiffeomorphism $\sigma:\left(\mathbf{R}^{*},\left.R^{1 \mid 1}\right|_{\mathbf{R}^{*}}\right) \rightarrow\left(\mathbf{R}^{*},\left.R^{1 \mid 1}\right|_{\mathbf{R}^{*}}\right)$ is given by

$$
\sigma^{\#} x=1 / x \text { and } \sigma^{\#} \theta=-(1 / x)^{2} \theta \text {. }
$$

1.4. Example. $\mathfrak{G}_{(m+n)^{2}}(\mathrm{GL}(m+n))=\left(\mathrm{GL}\left(V_{0} \oplus V_{1}\right), \mathrm{GL}_{s}(m+n \mid m+n)\right)$, the general linear supergroup of transformations of the $(m, n)$-dimensional supervector space $V_{0} \oplus V_{1}$. (We shall use the notation $\mathrm{GL}_{s}(m \mid n)$, or $\mathrm{GL}_{s}\left(V_{0} \mid V_{1}\right)$, to distinguish this supergroup from the more common $\mathrm{GL}(m \mid n)$; see Example 1.5 below and compare with [2].)

Recall that each $(m, n)$-dimensional supervector space $V=V_{0} \oplus V_{1}$ gives rise to the $(m, n)$-dimensional affine supermanifold $S V=\left(V_{0},\left(\left.C^{\infty}\right|_{V_{0}}\right) \otimes \wedge\left(V_{1}^{*}\right)\right)$ (cf. [2-4]). The supermanifoldification of the supervector space $V=V_{0} \oplus V_{1}$ is, on the other hand, the $(m+n, m+n)$-dimensional affine supermanifold $V_{s}:=S(V \oplus \Pi V)$, where $\Pi$ is the change of parity functor of Berezin and Leites (see [2, 4, 6]). This notion has proved to be useful while working on supervector bundles and superlinear actions (see [11, 12] for details). In particular, $\operatorname{Hom}(V, V)_{s}$ is the affine supermanifold of dimension $\left((m+n)^{2}\right.$, $\left.(m+n)^{2}\right)$ whose underlying smooth manifold is $\operatorname{Hom}(V, V)$. Following [12], we can introduce even and odd linear coordinates $\left(\left\{A^{b j}, \pi \Gamma^{b J}, \pi \Theta^{B j}, D^{B J}\right\}\right.$ and $\left\{\pi A^{b j}, \Gamma^{b J}, \Theta^{B j}, \pi D^{B J}\right\}$, respectively) on it, arrange them in matrix form,

$$
\left(\begin{array}{cc}
A^{b j}+\pi A^{b j} & \Gamma^{b J}+\pi \Gamma^{b J} \\
\Theta^{B j}+\pi \Theta^{B j} & D^{B J}+\pi D^{B J}
\end{array}\right),
$$

and define the map $\mu: \operatorname{Hom}(V, V)_{s} \times \operatorname{Hom}(V, V)_{s} \rightarrow \operatorname{Hom}(V, V)_{s}$ so as to obtain a functorial correspondence between matrix multiplication and composition of left supermodule morphisms (cf. [14]; see also [12]); thus,

$$
\begin{aligned}
& \left(\begin{array}{cc}
\mu^{\#}\left(A^{b j}+\pi A^{b j}\right) & \mu^{\#}\left(\Gamma^{b J}+\pi \Gamma^{b J}\right) \\
\mu^{\#}\left(\Theta^{B j}+\pi \Theta^{B j}\right) & \mu^{\#}\left(D^{B J}+\pi D^{B J}\right)
\end{array}\right) \\
& =\left(\begin{array}{cc}
p_{1}^{\#}\left(A^{b j}+\pi A^{b j}\right) & p_{1}^{\#}\left(\Gamma^{b J}+\pi \Gamma^{b J}\right) \\
p_{1}^{\#}\left(\Theta^{B j}+\pi \Theta^{B j}\right) & p_{1}^{\#}\left(D^{B J}+\pi D^{B J}\right)
\end{array}\right) \\
& \quad \times\left(\begin{array}{cc}
p_{2}^{\#}\left(A^{b j}+\pi A^{b j}\right) & p_{2}^{\#}\left(\Gamma^{b J}+\pi \Gamma^{b J}\right) \\
p_{2}^{\#}\left(\Theta^{B j}+\pi \Theta^{B j}\right) & p_{2}^{\#}\left(D^{B J}+\pi D^{B J}\right)
\end{array}\right)
\end{aligned}
$$

where $p_{i}: \operatorname{Hom}(V, V)_{s} \times \operatorname{Hom}(V, V)_{s} \rightarrow \operatorname{Hom}(V, V)_{s}$ denotes the projection morphism onto the $i$ th factor $(i=1,2)$.

Now, matrix (1) is invertible if and only if (cf. [2-4])

$$
\operatorname{det}\left(\begin{array}{cc}
A^{h j} & \pi \Gamma^{b J} \\
\pi \Theta^{B j} & D^{B J}
\end{array}\right)^{\sim} \neq 0 .
$$


This condition defines the open subset $\mathrm{GL}(V)=\mathrm{GL}\left(V_{0} \oplus V_{1}\right)$ of $\operatorname{Hom}(V, V)$. Therefore, the same condition picks up an open subsupermanifold of $\operatorname{Hom}(V, V)_{s}$; such supermanifold is, by definition, $\mathrm{GL}_{s}\left(V_{0} \mid V_{1}\right)$; its structural sheaf, $\mathrm{GL}_{s}(m+n \mid m+n)$, is $\left.R^{(m+n)^{2} \mid(m+n)^{2}}\right|_{\mathrm{GL}(m+n)}$. By restricting the morphism $\mu$ above to such a supermanifold and defining the inverse morphism $\sigma$ in terms of the given coordinates so as to obtain the inverse matrix of (1), $\mathrm{GL}_{s}\left(V_{0} \mid V_{1}\right)$ becomes a Lie supergroup. Note that Example 1.3 above corresponds to the special case $\mathrm{GL}_{s}(1 \mid 0)$.

1.5. Example. $\mathfrak{G}_{2 m n}(\mathrm{GL}(m) \times \mathrm{GL}(n))=\left(\mathrm{GL}\left(V_{0}\right) \times \mathrm{GL}\left(V_{1}\right), \mathrm{GL}(m \mid n)\right)$, also denoted by $\mathrm{GL}(m \mid n)$, or $\mathrm{GL}\left(V_{0} \mid V_{1}\right), V=V_{0} \oplus V_{1}$ being an $(m, n)$ dimensional supervector space (cf. [2]).

For the supergroup of Example 1.4 above, the conditions

$$
\pi \Gamma^{b J}=0, \quad \pi \Theta^{B j}=0, \quad \pi A^{b j}=0, \quad \pi D^{B J}=0, \quad \operatorname{det}\left(A^{b j}\right) \operatorname{det}\left(D^{B J}\right) \neq 0
$$

define an embedded $\left(m^{2}+n^{2}, 2 m n\right)$-dimensional subsupermanifold of $\mathrm{GL}_{s}\left(V_{0} \mid V_{1}\right)$. The restriction of the same morphisms $\mu$ and $\sigma$ and the same identity element make this subsupermanifold into a Lie supergroup.

1.6. Definition. Let $\left(G, A_{G}\right)$ be a Lie supergroup and let $\mu_{G}, \mathscr{E}_{G}, \sigma_{G}$ be its multiplication, identity, and inversion morphisms, respectively. Let $\left(H, A_{H}\right)$ be an immersed (resp. embedded) subsupermanifold of $\left(G, A_{G}\right)$ and let $i$ : $\left(H, A_{H}\right) \rightarrow\left(G, A_{G}\right)$ be the corresponding immersion (resp. embedding). Then $\left(H, A_{H}\right)$ is a Lie subsupergroup of $\left(G, A_{G}\right)$ if $\left(H, A_{H}\right)$ is a Lie supergroup itself and $i$ is a homomorphism (cf. [5] and [15]); that is, if $\mu_{H}, \mathscr{C}_{H}, \sigma_{H}$ are the multiplication, identity, and inversion morphisms of $\left(H, A_{H}\right)$, then

$$
\mu_{G} \circ\left(i \circ \pi_{1} \times i \circ \pi_{2}\right)=i \circ \mu_{H},
$$

regarded as morphisms from $\left(H, A_{H}\right) \times\left(H, A_{H}\right)$ into $\left(G, A_{G}\right)$. As usual, $\pi_{i}$ denotes the projection of the product $\left(H, A_{H}\right) \times\left(H, A_{H}\right)$ into the $i$ th factor.

1.7. Remark. Just as in the theory of Lie groups, from the single condition above one obtains

$$
\mathscr{E}_{G} \circ i=i \circ \mathscr{E}_{H} \text { and } \sigma_{G} \circ i=i \circ \sigma_{H} .
$$

The proof of these properties follows easily from the following two lemmas, which we shall have occasion to use again in this work.

1.8. Lemma. Let $\left(M, A_{M}\right)$ be a supermanifold and let $p \in M$ be an arbitrary point. Let $\mathscr{E}_{p}:\left(M, A_{M}\right) \rightarrow\left(M, A_{M}\right)$ be the composition $\delta_{p} \circ C_{\left(M, A_{M}\right)}$. Then, for any morphism $\alpha:\left(M, A_{M}\right) \rightarrow\left(M, A_{M}\right)$,

$$
\alpha \circ \mathscr{E}_{p}=\mathscr{E}_{\dot{\alpha}(p)} \text { and } \mathscr{E}_{p} \circ \alpha=\mathscr{E}_{p} .
$$

Proof. Note, on the one hand, that for any $f \in A_{M}(M)$ and for any $q \in M$ we have $\mathscr{E}_{q}^{\#} f=\tilde{f}(q) 1_{A(M)}$. On the other hand, $\left(\alpha^{\#} f\right)^{\sim}(p)=\tilde{f}(\tilde{\alpha}(p))$ (cf. [4]). Hence,

$$
\left(\mathscr{E}_{p} \circ \alpha\right)^{\#} f=\mathscr{E}_{p}^{\#}\left(\alpha^{\#} f\right)=\tilde{f}(\tilde{\alpha}(p)) 1_{A(M)}=\mathscr{E}_{\tilde{\alpha}(p)}^{\#} f .
$$


Similarly, since $\alpha^{\#}: A_{M}(M) \rightarrow A_{M}(M)$ is a morphism of $\mathbf{R}$-superalgebras, we have

$$
\left(\mathscr{E}_{p} \circ \alpha\right)^{\#} f=\alpha^{\#}\left(\mathscr{E}_{p}^{\#} f\right)=\alpha^{\#}\left(\tilde{f}(p) 1_{A(M)}\right)=\tilde{f}(p) 1_{A(M)}=\mathscr{E}_{p}^{\#} f .
$$

This verifies that the effect of $\left(\alpha \circ \mathscr{E}_{p}\right)^{\#}$ (resp. $\left.\left(\mathscr{E}_{p} \circ \alpha\right)^{\#}\right)$ on the superalgebra $A_{M}(M)$ of global sections is the same as that of $\mathscr{E}_{\dot{\alpha}(p)}^{\#}\left(\right.$ resp. $\left.\mathscr{E}_{p}^{\#}\right)$. Therefore, the morphisms are the same.

1.9. Lemma. Let $\left(G, A_{G}\right)$ be a Lie supergroup and let $\mu$ be its composition morphism. Then $\mu$ is an epimorphism. More generally, the following cancellation law holds true: for any morphisms $\alpha, \beta, \gamma:\left(G, A_{G}\right) \rightarrow\left(G, A_{G}\right)$,

$$
\mu \circ(\alpha \times \beta)=\mu \circ(\gamma \times \beta) \Rightarrow \alpha=\gamma \text { and } \mu \circ(\alpha \times \beta)=\mu \circ(\alpha \times \gamma) \Rightarrow \beta=\gamma \text {. }
$$

Proof. Let $\sigma$ and $\mathscr{E}_{e}$ be the inversion and identity morphisms of the supergroup. Assume that $\mu \circ(\alpha \times \beta)=\mu \circ(\gamma \times \beta)$, and consider the composite

$$
\left(G, A_{G}\right) \stackrel{\lambda}{\rightarrow}\left(G, A_{G}\right) \times\left(G, A_{G}\right) \stackrel{\mu}{\rightarrow}\left(G, A_{G}\right)
$$

where $\lambda=\mu \circ(\alpha \times \beta) \times \sigma \circ \beta$. By hypothesis, $\lambda=\mu \circ(\gamma \times \beta) \times \sigma \circ \beta$. If we denote by $\pi_{i}$ the projection morphism of the product of three copies of $\left(G, A_{G}\right)$ onto the $i$ th factor, we have

$$
\begin{aligned}
\mu \circ( & \mu \circ(\alpha \times \beta) \times \sigma \circ \beta)=\mu \circ\left(\mu \circ\left(\pi_{1} \times \pi_{2}\right) \times \pi_{3}\right) \circ(\alpha \times \beta \times \sigma \circ \beta) \\
& =\mu \circ\left(\pi_{1} \times \mu \circ\left(\pi_{2} \times \pi_{3}\right)\right) \circ(\alpha \times \beta \times \sigma \circ \beta) \\
& =\mu \circ\left(\pi_{1} \times \mu \circ(\mathrm{id} \times \sigma)\right) \circ(\alpha \times \beta \times \beta) \\
& =\mu \circ\left(\pi_{1} \times \mathscr{E}_{e}\right) \circ(\alpha \times \beta \times \beta)=\pi_{1} \circ(\alpha \times \beta \times \beta)=\alpha .
\end{aligned}
$$

But the same string of equalities, with $\alpha$ replaced by $\gamma$, shows that

$$
\mu \circ(\mu \circ(\gamma \times \beta) \times \sigma \circ \beta)=\gamma .
$$

Therefore, $\mu \circ(\alpha \times \beta)=\mu \circ(\gamma \times \beta) \Rightarrow \alpha=\gamma$. The other cancellation law is proved similarly.

1.10. Remark. Another point is worth observing from Definition 1.6. The definition, as it stands, allows the irrational flow on the torus $(H \simeq \mathbf{R}$ and $\left.G=S^{1} \times S^{1}\right)$ to be the underlying manifold of a Lie subsupergroup. This degree of generality, on the other hand, keeps the morphism $i:\left(H, A_{H}\right) \rightarrow\left(G, A_{G}\right)$ from being a monomorphism (i.e., a left cancelable morphism). It follows, however, in a straightfoward manner that $i$ will be a monomorphism if and only if $\left(H, A_{H}\right)$ is an embedded subsupermanifold of $\left(G, A_{G}\right)$, which is true if and only if $H$ is a closed Lie subgroup of $G$ and $i^{\#}: A_{G} \rightarrow \tilde{i}_{*} A_{H}$ is an epimorphism; $\tilde{i}$ being the embedding of $H$ into $G$.

As pointed out to us by $\mathrm{R}$. Berlanga, this raises the question of classifying the different subsupergroup structures $i:\left(H, A_{H}\right) \rightarrow\left(G, A_{G}\right)$ that a given closed subgroup $H$ of $G$ can support. We shall deal with the classification problem elsewhere. Observe, however, that there can be several different such structures. 
In fact, taking $\left(G, A_{G}\right)=\left(\mathbf{R}^{m}, R^{m \mid n}\right)$ and $H=\{e\} \simeq\{*\}$ from Example 1.2 above, there are $n$ different additive subsupergroup structures supported at $H$; they are classified by their odd dimension.

\section{LEFT AND RIGHT INVARIANCE ON LIE SUPERGROUPS}

2.1. Let $\left(G, A_{G}\right)$ be a Lie supergroup. For each point $g \in G$, we define left and right translations by $g$ as the supermanifold morphisms

$$
L_{g}:=\mu \circ\left(\mathscr{E}_{g} \times \mathrm{id}\right) \text { and } R_{g}:=\mu \circ\left(\mathrm{id} \times \mathscr{E}_{g}\right),
$$

respectively. Here $\mathscr{E}_{g}:\left(G, A_{G}\right) \rightarrow\left(G, A_{G}\right)$ denotes the aorphism $\delta_{g} \circ C_{\left(G, A_{(i}\right)}$, whose corresponding superalgebra morphism $\mathscr{E}_{g}^{\#}: A_{G}(U) \rightarrow A_{G}\left(\tilde{\mathscr{E}}_{g}^{-1}(U)\right)$ is

$$
\mathscr{E}_{g}^{\#} f= \begin{cases}\tilde{f}(g) 1_{A_{G}\left(\tilde{\mathscr{E}}_{g-1}(U)\right)} & \text { if } g \in U, \\ 0 & \text { if } g \notin U .\end{cases}
$$

2.2. Proposition. The morphisms $L_{g}$ and $R_{g}$ are superdiffeomorphisms whose inverses are respectively given by

$$
\left(L_{g}\right)^{-1}=L_{g^{-1}} \text { and }\left(R_{g}\right)^{-1}=R_{g^{-1}} .
$$

Proof. We shall prove here only that $\left(R_{g}\right)^{-1}=R_{g^{-1}}$; that is,

(3) $\mu \circ\left(\mathrm{id} \times \mathscr{E}_{g^{-1}}\right) \circ \mu \circ\left(\mathrm{id} \times \mathscr{E}_{g}\right)=\mathrm{id}$ and $\mu \circ\left(\mathrm{id} \times \mathscr{E}_{g}\right) \circ \mu \circ\left(\mathrm{id} \times \mathscr{E}_{g^{-1}}\right)=\mathrm{id}$ as morphisms from $\left(G, A_{G}\right)$ into itself. We start by making use of the definition of the product; thus,

$$
\begin{aligned}
\mu \circ\left(\mathrm{id} \times \mathscr{E}_{g^{-1}}\right) \circ \mu \circ\left(\mathrm{id} \times \mathscr{E}_{g}\right) & =\mu \circ\left(\mathrm{id} \circ\left[\mu \circ\left(\mathrm{id} \times \mathscr{E}_{g}\right)\right] \times \mathscr{E}_{g^{-1}} \circ\left[\mu\left(\mathrm{id} \times \mathscr{E}_{g}\right)\right]\right) \\
& =\mu \circ\left(\left[\mu \circ\left(\mathrm{id} \times \mathscr{E}_{g}\right)\right] \times \mathscr{E}_{g^{-1}} \circ \mu \circ\left(\mathrm{id} \times \mathscr{E}_{g}\right)\right) .
\end{aligned}
$$

It then follows from Lemma 1.8 that

$$
\mathscr{E}_{g}^{-1} \circ \mu \circ\left(\mathrm{id} \times \mathscr{E}_{g}\right)=\mathscr{E}_{g^{-1}}
$$

Therefore,

$$
\begin{aligned}
\mu \circ\left(\mathrm{id} \times \mathscr{E}_{g^{-1}}\right) \circ \mu \circ\left(\mathrm{id} \times \mathscr{E}_{g}\right) & =\mu \circ\left(\left[\mu \circ\left(\mathrm{id} \times \mathscr{E}_{g}\right)\right] \times \mathscr{E}_{g^{-1}}\right) \\
& =\mu \circ\left(\mu \circ\left(\pi_{1} \times \pi_{2}\right) \times \pi_{3}\right) \circ\left(\mathrm{id} \times \mathscr{E}_{g} \times \mathscr{E}_{g^{-1}}\right) \\
& =\mu \circ\left(\pi_{1} \times \mu \circ\left(\pi_{2} \times \pi_{3}\right)\right) \circ\left(\mathrm{id} \times \mathscr{E}_{g} \times \mathscr{E}_{g^{-1}}\right) \\
& =\mu \circ\left(\mathrm{id} \times \mu \circ\left(\mathscr{E}_{g} \times \mathscr{E}_{g^{-1}}\right)\right),
\end{aligned}
$$

where we have used the definition of the product and the associativity property of $\mu$. Since $\tilde{\sigma}(g)=g^{-1}$, it follows from Lemma 1.8 again that

$$
\mathscr{E}_{g^{-1}}=\sigma \circ \mathscr{E}_{g} \text {. }
$$

Hence

$$
\begin{aligned}
\mu \circ\left(\mathrm{id} \times \mathscr{E}_{g^{-1}}\right) \circ \mu \circ\left(\mathrm{id} \times \mathscr{E}_{g}\right) & =\mu \circ\left(\mathrm{id} \times \mu \circ\left(\mathscr{E}_{g} \times \mathscr{E}^{g^{-1}}\right)\right) \\
& =\mu \circ\left(\mathrm{id} \times \mu \circ(\mathrm{id} \times \sigma) \circ \mathscr{E}_{g}\right) \\
& =\mu \circ\left(\mathrm{id} \times \mathscr{E}_{e} \circ \mathscr{E}_{g}\right) \\
& =\mu \circ\left(\mathrm{id} \times \mathscr{E}_{e}\right)=\mathrm{id},
\end{aligned}
$$


where use has been made of the inverse property for $\sigma$ and of Lemma 1.8 to conclude that $\mathscr{E}_{e} \circ \mathscr{E}_{g}=\mathscr{E}_{e}$. In a similar manner one proves that $R_{g^{-1}}$ is a right inverse, too. Thus, $\left(R_{g}\right)^{-1}=R_{g^{-1}}$.

2.3. Proposition. Let $\left(G, A_{G}\right)$ be a Lie supergroup and let $L_{g}$ and $R_{g}$ be the left and right translations by $g \in G$, as in 2.1(1). Then for any other $h \in G$,

$$
L_{g} \circ L_{h}=L_{g h} \text { and } R_{g} \circ R_{h}=R_{h g} .
$$

Proof. This is a straightforward consequence of the definitions and Lemma 1.8. In fact,

$$
\begin{aligned}
R_{g} \circ R_{h} & =\mu \circ\left(\mathrm{id} \times \mathscr{E}_{g}\right) \circ \mu \circ\left(\mathrm{id} \times \mathscr{E}_{h}\right)=\mu \circ\left(\mu \circ\left(\mathrm{id} \times \mathscr{E}_{h}\right) \times \mathscr{E}_{g} \circ \mu \circ\left(\mathrm{id} \times \mathscr{E}_{h}\right)\right) \\
& =\mu \circ\left(\mu \circ\left(\mathrm{id} \times \mathscr{E}_{h}\right) \times \mathscr{E}_{g}\right)=\mu \circ\left(\mathrm{id} \times \mu \circ\left(\mathscr{E}_{h} \times \mathscr{E}_{g}\right)\right)=\mu \circ\left(\mathrm{id} \times \mathscr{E}_{\tilde{\mu}(h, g)}\right) \\
& =\mu \circ\left(\mathrm{id} \times \mathscr{E}_{h g}\right)=R_{h g} .
\end{aligned}
$$

The corresponding property for left translations is similarly verified.

2.4. In what follows we shall be concerned with left invariant supervector fields on $\left(G, A_{G}\right)$. We shall show that their characterization is exactly the same as in the smooth theory. Let $\operatorname{Der} A_{G}$ be the sheaf (over $G$ ) of superderivations of the structural sheaf $A_{G}$. Let $\operatorname{Der} A_{G}(G)$ be its corresponding superspace of global sections. Recall that $\operatorname{Der} A_{G}(G)$ is the real subsupervector space of End $A_{G}(G)$,

$$
\operatorname{Der} A_{G}(G)=\left(\operatorname{Der} A_{G}(G)\right)_{0} \oplus\left(\operatorname{Der} A_{G}(G)\right)_{1},
$$

where

$\left(\operatorname{Der} A_{G}(G)\right)_{\mu}=\left\{X \in \operatorname{End} A_{G}(G) \mid\left(\forall f, g \in A_{G}(G) ; f\right.\right.$ homogeneous $)$

$$
\left.X(f g)=X(f) g+(-1)^{|f| \mu} f X(g)\right\} .
$$

The supervector space $\operatorname{Der} A_{G}(G)$ inherits from End $A_{G}(G)$ a Lie superalgebra structure, the Lie superbracket of which is given on homogeneous elements $X$ and $Y$ by

$$
[X, Y]=X \circ Y-(-1)^{|X||Y|} Y \circ X .
$$

2.5. Definition. A superderivation, $X \in \operatorname{Der} A_{G}(G)$, is left invariant if for each $g \in G, L_{g^{*}} X=X$ (compare with [2-5]), where

$$
L_{g^{*}} X=\left(L_{g^{-1}}\right)^{\#} \circ X \circ\left(L_{g}\right)^{\#} .
$$

2.6. Remark. The map $g \mapsto L_{g^{*}}$ defines a representation of the Lie group $G$ on $\operatorname{Der} A_{G}(G)$ acting via automorphisms of the Lie superalgebra structure. In particular, the subsuperspace consisting of left invariant superderivations is itself a Lie superalgebra. It will be denoted by $\mathfrak{g}\left(=\mathfrak{g}_{0} \oplus \mathfrak{g}_{1}\right)$. 
2.7. Proposition. There exists a supervector space isomorphism

$$
\mathfrak{g}=\mathfrak{g}_{0} \oplus \mathfrak{g}_{1} \rightarrow(\mathrm{ST})_{e}\left(G, A_{G}\right)=\left\{(\mathrm{ST})_{e}\left(G, A_{G}\right)\right\}_{0} \oplus\left\{(\mathrm{ST})_{e}\left(G, A_{G}\right)\right\}_{1},
$$

where, $\left\{(\mathrm{ST})_{e}\left(G, A_{G}\right)\right\}_{\mu}$ is the space of germs at e of homogeneous superderivations of degree $\mu$ of the superalgebra $A_{G}(U)$, with $U \ni e$.

Proof. The isomorphism is given by assigning, to each left invariant superderivation, its germ at the identity. Let us verify first that this prescription yields a surjection. Let $\xi \in(\mathrm{ST})_{e}\left(G, A_{G}\right)$ be arbitrary and consider, for each $g \in G$, the following morphism induced on the stalk of $A_{G}$ over $g$ :

$$
\left(A_{G}\right)_{g} \stackrel{\left(L_{g}\right)_{g}^{*}}{\rightarrow}\left(A_{G}\right)_{e} \stackrel{\xi}{\rightarrow}\left(A_{G}\right)_{e} \stackrel{\left(L_{g}-1\right)_{e}^{*}}{\rightarrow}\left(A_{G}\right)_{g} .
$$

Then define the section $g \mapsto \widehat{\xi}_{g}=\left(L_{g^{-1}}\right)_{e}^{\#} \circ \xi \circ\left(L_{g}\right)_{g}^{\#}$ of the sheaf space, LDer $A_{G}$, associated to the presheaf Der $A_{G}$ (cf. [16]). Since $\operatorname{Der} A_{G}$ is in fact a sheaf, we have

$$
\operatorname{Der} A_{G}(\cdot) \simeq \Gamma\left(\cdot, \mathbf{L} \operatorname{Der} A_{G}\right)
$$

and therefore $g \mapsto \widehat{\xi}_{g}$ defines a global section, $\widehat{\xi} \in \operatorname{Der} A_{G}(G)$. We shall now show that this section is left invariant. In fact, for each $h \in G$,

$$
\begin{aligned}
\left(L_{h^{*}} \widehat{\xi}\right)_{g} & =\left\{\left(L_{h^{-1}}\right)^{\#} \circ \widehat{\xi}_{0} \circ\left(L_{h}\right)^{\#}\right\}_{g}=\left(L_{h^{-1}}\right)_{h^{-1} g}^{\#} \circ \widehat{\xi}_{h^{-1}} \circ\left(L_{h}\right)_{g}^{\#} \\
& =\left(L_{h^{-1}}\right)_{h^{-1} g}^{\#} \circ\left(L_{\left(h^{-1} g\right)^{-1}}\right)_{e}^{\#} \circ \xi \circ\left(L_{h^{-1}}\right)_{h^{-1} g}^{\#} \circ\left(L_{h}\right)_{g}^{\#} \\
& =\left(L_{\left(h^{-1} g\right)^{-1}}\right)_{e}^{\#} \circ \xi \circ\left(L_{h} \circ L_{h^{-1}}\right)_{g}^{\#} \\
& =\left(L_{g^{-1}}\right)_{e}^{\#} \circ \xi \circ\left(L_{g}\right)_{g}^{\#}=\widehat{\xi}_{g},
\end{aligned}
$$

where use has been made of the contravariance of \#, and the multiplicativity property of left translations given in Proposition 2.3.

Finally, to prove that the morphism $X \mapsto X_{e}$ is injective, note that if $X_{e}=$ $Y_{e}$, then $X$ and $Y$ coincide in some open neighborhood of the identity. By left invariance, they coincide everywhere (this means that we cover $G$ by open subsets which are left translates of an open neighborhood of $e$, over each of which the restrictions of $X$ and $Y$ coincide; since $\operatorname{Der} A_{G}$ is a sheaf, $X=$ $Y)$.

2.8. Remark. It has been proved in [ 3 and 4$]$ that for any $(m, n)$-dimensional supermanifold $\left(M, A_{M}\right)$, the sheaf $\operatorname{Der} A_{M}$ is a locally free sheaf of $A_{M^{-}}$ modules over $M$, with $m$ even generators and $n$ odd. Hence, for any point $p \in$ $M$, the supervector space of superderivations at $p$ is an $(m, n)$-dimensional supervector space. In particular, $(\mathrm{ST})_{\mathcal{e}}\left(G, A_{G}\right)$, and hence $\mathfrak{g}$ is a finitedimensional supervector space whose dimension is precisely that of the supergroup.

More important, via the isomorphism given in Proposition 2.7, one can define a global frame on the supermanifold $\left(G, A_{G}\right)$ consisting entirely of 
left invariant supervector fields, namely by choosing any homogeneous basis $\left\{\xi_{1}, \ldots, \xi_{r} ; \zeta_{1}, \ldots, \zeta_{s}\right\}$ of $(\mathrm{ST})_{e}\left(G, A_{G}\right)$ and looking at their corresponding left invariant superderivations. Thus, we obtain the following.

2.9. Proposition. $\operatorname{Der} A_{G}(G) \simeq A_{G}(G) \otimes \mathfrak{g}$, and therefore the supertangent bundle $\left(G, A_{G}\right)$, is trivial; that is,

$$
\left(\mathrm{ST} G, \mathrm{ST} A_{G}\right) \simeq\left(G, A_{G}\right) \times \mathfrak{g}_{s},
$$

where $\mathfrak{g}_{s}$ is the supermanifoldification of the Lie algebra $\mathfrak{g}=\mathfrak{g}_{0} \oplus \mathfrak{g}_{1}$ (cf. [11] and Example 1.4 above).

Proof. We include here a brief argument, based on the approach of [11], that proves that a frame over some open subset $U \subset G$ yields a trivialization of the supertangent bundle over the same open subset (and hence the statement follows). Specifically, we shall see how giving such a frame is the same as giving an isomorphism $\varphi_{U}$ which makes the following diagram commute:

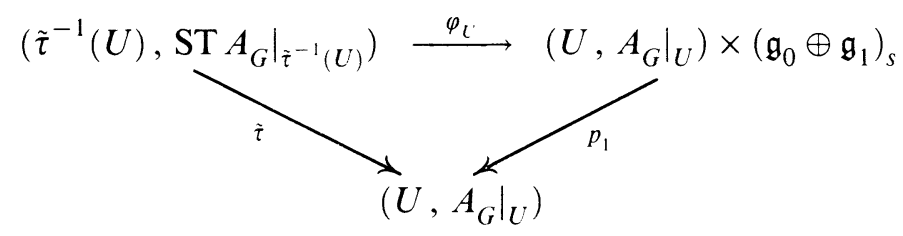

where $\tilde{\tau}$ denotes the submersion morphism of the supertangent bundle into the base supermanifold and $p_{1}$ the projection morphism of the product onto the first factor. Now, vector bundles were approached in [11] in such a way that supermanifold morphisms $\sigma:\left(U,\left.A_{G}\right|_{U}\right) \rightarrow\left(\tilde{\tau}^{-1}(U)\right.$, ST $\left.\left.A_{G}\right|_{\tilde{\tau}^{-1}(U)}\right)$, satisfying $\tilde{\tau} \circ \sigma=\mathrm{id}:\left(U,\left.A_{G}\right|_{U}\right) \rightarrow\left(U,\left.A_{G}\right|_{U}\right)$, correspond in a one-to-one fashion with sections $\sigma \in \operatorname{Der} A_{G}(U)$. If $\left\{\xi_{1}, \ldots, \xi_{r} ; \zeta_{1}, \ldots, \zeta_{s}\right\}$ is a graded frame over $U$, with $\left|\xi_{i}\right|=0$ and $\left|\zeta_{\mu}\right|=1$, each $\sigma \in \operatorname{Der} A_{G}(U)$ can be written uniquely in the form $\sigma=\sum f^{i} \xi_{i}+\sum \varphi^{\mu} \zeta_{\mu}$ where $f^{i}, \varphi^{\mu} \in A_{G}(U)$. That is, $\sigma$ is uniquely characterized by the order $(r+s)$-tuple $\left\{f^{1}, \ldots, f^{r} ; \varphi^{1}, \ldots, \varphi^{s}\right\} \in$ $A_{G}(U)^{r} \oplus A_{G}(U)^{s}$. The key point observed in [11] is that $A_{G}(U)^{r} \oplus A_{G}(U)^{s}$ is isomorphic to the set of all supermanifold morphisms from $\left(U,\left.A_{G}\right|_{U}\right)$ into the supermanifoldification of any $(r, s)$-dimensional supervector space. That is,

$$
A_{G}(U)^{r} \oplus A_{G}(U)^{s} \simeq \operatorname{Mor}\left(\left(U,\left.A_{G}\right|_{U}\right),\left(\mathfrak{g}_{0} \oplus \mathfrak{g}_{1}\right)_{s}\right) .
$$

Thus, if we denote by $\Psi^{\sigma}$ the morphism $\left(U,\left.A_{G}\right|_{U}\right) \rightarrow\left(\mathfrak{g}_{0} \oplus \mathfrak{g}_{1}\right)_{s}$ that corresponds to the $(r+s)$-tuple $\left\{f^{1}, \ldots, f^{r} ; \varphi^{1}, \ldots, \varphi^{s}\right\} \in A_{G}(U)^{r} \oplus A_{G}(U)^{s}$ that $\sigma$ gives rise to, then the trivialization $\varphi_{U}$ is uniquely determined by the pair of conditions id $=p_{1} \circ \varphi_{U} \circ \sigma$ and $\Psi^{\sigma}=p_{1} \circ \varphi_{U} \circ \sigma, \forall \sigma \in \operatorname{Der} A_{G}(U)$.

2.10. Corollary. Let $\left(G, A_{G}\right)$ be a Lie supergroup and let $\mathfrak{g}=\mathfrak{g}_{0} \oplus \mathfrak{g}_{1}$ be its Lie superalgebra. Let $\mathfrak{g}_{1}^{*}$ be the vector space dual of $\mathfrak{g}_{1}$. Then

$$
A_{G}(G) \simeq C^{\infty}(G) \otimes \bigwedge\left(\mathfrak{g}_{1}^{*}\right) .
$$


Proof. The structural sheaf $A_{G}$ of a supermanifold $\left(G, A_{G}\right)$ is, by definition, locally isomorphic to the sheaf $\operatorname{Gr} A_{G}$, where (cf. [7])

$$
\operatorname{Gr} A_{G}=\bigoplus_{k \geq 0} \mathrm{Gr}^{k} \quad \mathrm{Gr}^{k} A_{G}=J_{G}^{k} / J_{G}^{k+1},
$$

and $A_{G}=J_{G}^{0} \supset J_{G}^{1} \supset J_{G}^{2} \supset \cdots J_{G}^{k} \supset \cdots$ is the $J_{G}$-adic filtration of $A_{G}$ define by the sheaf of ideals over $G, J_{G}=\left(\left(A_{G}\right)_{1}\right)$, generated by the odd subsheaf $\left(A_{G}\right)_{1}$. Each $\mathrm{Gr}^{k} A_{G}$ is a sheaf of $\mathrm{Gr}^{0} A_{G}$-modules and, when viewed as a sheaf of $\mathrm{Gr}^{0} A_{G}$-algebras, $\operatorname{Gr} A_{G}$ is generated by $\mathrm{Gr}^{1} A_{G}$. Since $A_{M}$ is supercommutative, $\operatorname{Gr} A_{M}$ is a homomorphic image of the sheaf $\bigwedge_{\mathrm{Gr}^{0}{ }_{A}} \mathrm{Gr}^{1} A_{G}$. It is isomorphic to it only if $\mathrm{Gr}^{1} A_{G}$ is finitely generated over $\mathrm{Gr}^{0} A_{G}$, i.e., if the odd dimension of the supermanifold is finite (recall that, by definition, the sheaf $\mathrm{Gr}^{0} A_{G}$ is isomorphic to the sheaf $C_{G}^{\infty}$ of real smooth functions over $G$ and $\mathrm{Gr}^{1} A_{G}$ is a locally free sheaf of $\mathrm{Gr}^{0} A_{G}$-modules over $G$ whose rank is precisely the odd dimension of the supermanifold; cf [7]).

Now the stalk at any $x \in G\left(\mathrm{Gr}^{1} A_{G}\right)_{x}=J_{G, x} / J_{G, x}^{2}$, can be identified with $\left(\mathscr{M}_{x} / \mathscr{M}_{x}^{2}\right)_{1}$, where $\mathscr{M}_{x}$ is the maximal ideal of $A_{G, x}$ consisting of all germs vanishing at $x$. This follows, since $\left(\mathscr{M}_{x}\right)_{1}=\left(J_{G, x}\right)_{1}$ and the even subspace of $J_{G, x}$ is $J_{G, x}^{2}$. In other words, $\left(\operatorname{Gr}^{1} A_{G}\right)_{x} \simeq\left(\operatorname{Hom}\left(\operatorname{Der} A_{G}, A_{G}\right)_{x}\right)_{1}$. Since $A_{G, x} \simeq C_{G, x}^{\infty} \oplus J_{G, x}$, it follows that when $\operatorname{Der} A_{G}(G) \simeq A_{G}(G) \otimes \mathfrak{g},\left(\mathrm{Gr}^{1} A_{G}\right)_{x} \simeq$ $C_{G, x}^{\infty} \otimes \mathfrak{g}_{1}^{*}$.

2.11. Remark. Once the triviality of the supertangent bundle of a Lie supergroup $\left(G, A_{G}\right)$ is settled, one may argue as in [4] to conclude that

$$
C^{\infty}(G) \simeq\left\{f \in A_{G}(U) \mid(\forall \mu=1, \ldots, s) \zeta_{\mu} f=0\right\}
$$

and

$$
\bigwedge\left(\mathfrak{g}_{1}^{*}\right) \simeq\left\{f \in A_{G}(U) \mid(\forall i=1, \ldots, r) \xi_{i} f=0\right\} .
$$

In fact, let $\left\{\xi_{1}, \ldots, \xi_{r} ; \zeta_{1}, \ldots, \zeta_{s}\right\}$ be the basis of $\mathfrak{g}$ obtained via Proposition 2.7 from the germs at $e \in G$ of the set $\left\{\partial_{z^{1}}, \ldots, \partial_{z^{r}} ; \partial_{\eta^{1}}, \ldots, \partial_{\eta^{s}}\right\}$ of superderivations of the superalgebra $A_{G}(U)$, with $\left(U,\left\{z^{1}, \ldots, z^{r} ; \eta^{1}, \ldots, \eta^{s}\right\}\right)$ a coordinate neighborhood around $e$. Then propagate the supermanifold structure over $U$, which is already given locally by definition, to the entire group via left translations. $G$ is thus covered by the family of open subsets $\left\{L_{g}(U): g \in\right.$ $G\}$, and an easy sheaf-theoretical argument then proves (11).

\section{LIE SUPERGROUP ACTIONS ON SUPERMANIFOLDS}

3.1. Definition. Let $\left(G, A_{G}\right)$ be a Lie supergroup and let $\left(M, A_{M}\right)$ be a supermanifold. $\left(G, A_{G}\right)$ acts on $\left(M, A_{M}\right)$ from the left if there is a supermanifold morphism

$$
\psi:\left(G, A_{G}\right) \times\left(M, A_{M}\right) \rightarrow\left(M, A_{M}\right)
$$


satisfying the following two identities (cf. [2, 5, 15]):

$$
\psi \circ\left(\pi_{1} \times \psi \circ\left(\pi_{2} \times \pi_{3}\right)\right)=\psi \circ\left(\mu \circ\left(\pi_{1} \times \pi_{2}\right) \times \pi_{3}\right)
$$

[both sides are morphisms $\left(G, A_{G}\right) \times\left(G, A_{G}\right) \times\left(M, A_{M}\right) \rightarrow\left(M, A_{M}\right)$ and this time the projections $\pi_{i}$ are defined on $\left.\left.\left(G, A_{G}\right) \times\left(G, A_{G}\right) \times(M, A)_{M}\right)\right]$ and

$$
\psi \circ\left(\mathscr{E}_{e} \circ \pi_{1} \times \pi_{2}\right)=\pi_{2}
$$

[as morphisms $\left(G, A_{G}\right) \times\left(M, A_{M}\right) \rightarrow\left(M, A_{M}\right)$ ].

3.2. Example. $\mathrm{GL}_{s}\left(V_{0} \mid V_{1}\right)$ acting on $V_{2}, V=V_{0} \oplus V_{1}$ being an $(m, n)$ dimensional supervector space $V_{s}$ its supermanifoldification (cf. [11, 12] and Example 1.4 above). It has been shown in [12] that there is a natural way of generalizing the morphism that evaluates linear maps on vectors to the category of supermanifolds. The restriction of that morphism to $\mathrm{GL}_{s}\left(V_{0} \mid V_{1}\right) \times V_{s}$ yields $\psi: \mathrm{GL}_{s}\left(V_{0} \mid V_{1}\right) \times V_{s} \rightarrow V_{s}$, which defines a left action of $\mathrm{GL}_{s}\left(V_{0} \mid V_{1}\right)$ on $V_{s}$ in the sense just defined. In fact, the work in [12] was developed so as to make this happen; we refer the reader there for details. An explicit expression for $\psi$ in terms of the (linear) even and odd coordinates in $\operatorname{Hom}(V, V)_{s}$ as in Example 1.4 above, $\left\{A^{b j}, \pi \Gamma^{b J}, \pi \Theta^{B j}, D^{B J}\right\}$ and $\left\{\pi A^{b j}, \Gamma^{b J}, \Theta^{B j}, \pi D^{B J}\right\}$, respectively, and the (linear) even and odd coordinates in $V_{s},\left\{x^{j}, \pi \xi^{J}\right\}$ and $\left\{\pi x^{j}, \xi^{J}\right\}$, respectively, is obtained by multiplying matrix (1) in Example 1.4 on the left of the column matrix whose rows are $x^{j}+\pi x^{j}, j=1, \ldots, \operatorname{dim} V_{0}$, and $\pi \xi^{J}+\xi^{J}, J=1, \ldots, \operatorname{dim} V_{1}$, in that order (see Example 5.1 below).

3.3. Example. $\mathrm{GL}(m \mid n)$ acting on $G_{k \mid h}\left(V^{m \mid n}\right)$, the Grassmannian supermanifold, of $(k, h)$-dimensional supervector subspaces of the $(m, n)$-dimensional supervector space $V^{m \mid n}=V_{0} \oplus V_{1}$ (cf. [6-9, 13]).

Manin has shown that there is a natural action of the Lie group $\operatorname{GL}(m) \times$ $\mathrm{GL}(n)$ on $G_{k \mid h}\left(V^{m \mid n}\right.$ ) (cf. [6-9]). But, more generally, it was emphasized in [13] that there is also a natural action of the Lie supergroup $\operatorname{GL}\left(V_{0} \mid V_{1}\right)$ (cf. Example 1.5 above). In fact, one may introduce local coordinates in $G_{k \mid h}\left(V^{m \mid n}\right)$ and arrange them in matrix form as in [6-9]:

$$
\left(\begin{array}{ll}
x & \xi \\
\zeta & y
\end{array}\right),
$$

where $x=\left(x^{i j}\right)$ and $y=\left(y^{a b}\right)$ are the even coordinates and $\xi=\left(\xi^{i b}\right)$ and $\zeta=\left(\zeta^{a j}\right)$ are the odd ones $(1 \leq i \leq m-k, 1 \leq j \leq k, 1 \leq a \leq n-h$, $1 \leq b \leq h)$. It is in terms of this set of local coordinates that the following element of $\mathrm{GL}\left(V_{0} \mid V_{1}\right)$, supercoset representative, can be defined:

$$
\left(\begin{array}{llll}
1 & x & 0 & \xi \\
0 & 1 & 0 & 0 \\
0 & \zeta & 1 & y \\
0 & 0 & 0 & 1
\end{array}\right)
$$


The action of the supergroup can be explicitly obtained (under the assumption that the transformation takes place within the same coordinate patch) by solving for the coordinates $x^{\prime}, y^{\prime}, \xi^{\prime}$, and $\zeta^{\prime}$ of the new supercoset representative in the equation (cf. [13]),

$$
\left(\begin{array}{llll}
a & b & \alpha & \beta \\
c & d & \gamma & \delta \\
\pi & \rho & p & r \\
\sigma & \tau & s & t
\end{array}\right)\left(\begin{array}{llll}
1 & x & 0 & \xi \\
0 & 1 & 0 & 0 \\
0 & \zeta & 1 & y \\
0 & 0 & 0 & 1
\end{array}\right)=\left(\begin{array}{cccc}
1 & x^{\prime} & 0 & \xi^{\prime} \\
0 & 1 & 0 & 0 \\
0 & \zeta^{\prime} & 1 & y^{\prime} \\
0 & 0 & 0 & 1
\end{array}\right)\left(\begin{array}{cccc}
J & 0 & K & 0 \\
c & M & \gamma & T \\
U & 0 & H & 0 \\
\sigma & W & s & N
\end{array}\right)
$$

where the matrix on the left belongs to $\operatorname{GL}(m \mid n)$. There is a more succinct way of writing the action; the idea (suggested by $\mathrm{J}$. A. Wolf) is to conjugate both sides of this equation by the matrix that interchanges the second and third rows. The result can then be written as follows:

$$
Z^{\prime}=(A Z+B)(C Z+D)^{-1},
$$

where

$$
Z=\left(\begin{array}{ll}
x & \xi \\
\zeta & y
\end{array}\right) \text { and } Z^{\prime}=\left(\begin{array}{ll}
x^{\prime} & \xi^{\prime} \\
\zeta^{\prime} & y^{\prime}
\end{array}\right)
$$

and $A, B, C$, and $D$ are block matrices defined in terms of the entries of the original element in $\mathrm{GL}(m \mid n)$ as

$$
A=\left(\begin{array}{cc}
a & \alpha \\
\pi & p
\end{array}\right), \quad B=\left(\begin{array}{cc}
b & \beta \\
\rho & r
\end{array}\right), \quad C=\left(\begin{array}{cc}
c & \gamma \\
\sigma & s
\end{array}\right), \quad D=\left(\begin{array}{cc}
d & \delta \\
\tau & t
\end{array}\right) .
$$

Therefore, the action morphism is given by

$$
\psi^{\#} Z=\left\{\left(p_{i}^{\#} A\right)\left(p_{2}^{\#} Z\right)+\left(p_{1}^{\#} B\right)\right\}\left\{\left(p_{1}^{\#} C\right)\left(p_{2}^{\#} Z\right)+\left(p_{1}^{\#} D\right)\right\}^{-1},
$$

where the $p_{i}$ 's are the projection morphisms of the product supermanifold $\mathrm{GL}\left(V_{0} \mid V_{1}\right) \times G_{k \mid h}\left(V^{m \mid n}\right)$ (see also Example 5.2 below).

\section{THE ISOTROPY SUBSUPERGROUP OF AN ACTION}

4.1. Let $\left(G, A_{G}\right)$ be a Lie supergroup acting on the supermanifold $\left(M, A_{M}\right)$ via the morphism

$$
\psi:\left(G, A_{G}\right) \times\left(M, A_{M}\right) \rightarrow\left(M, A_{M}\right)
$$

and fix some point $p \in M$. Then $\psi$ induces a morphism

$$
\psi_{p}:\left(G, A_{G}\right) \rightarrow\left(M, A_{M}\right)
$$

by letting

$$
\psi_{p}:=\psi \circ\left(\mathrm{id} \times \delta_{p} \circ C_{\left(G, A_{i}\right)}\right)=\psi \circ\left(\mathrm{id} \times \mathscr{E}_{p}\right)
$$

where $\mathscr{E}_{p}:=\delta_{p} \circ C_{\left(G, A_{(i)}\right)}:\left(G, A_{G}\right) \rightarrow\left(M, A_{M}\right)$ is the supermanifold morphism whose corresponding map of presheaves is given by

$$
(\forall V \subset M, \text { open })\left(\forall f \in A_{M}(V)\right) \quad \mathscr{C}_{p}^{\#} f= \begin{cases}\tilde{f}(x) 1_{A_{t^{\prime}}(G)} & \text { if } x \in V, \\ 0 & \text { if } x \notin V .\end{cases}
$$


The underlying continuous maps, $\tilde{\psi}_{p}$ and $\tilde{\mathscr{E}}_{p}$, of $\psi_{p}$ and $\mathscr{E}_{p}$, respectively, are

$$
\tilde{\psi}_{p}: h \mapsto \tilde{\psi}(h, p) \text { and } \quad \tilde{\mathscr{E}}_{p}: h \mapsto p \quad \text { (constant map) }
$$

In a similar way, each point $g \in G$ gives rise to superdiffeomorphism

$$
\psi_{g}:\left(M, A_{M}\right) \rightarrow\left(M, A_{M}\right)
$$

defined by

$$
\psi_{g}:=\psi \circ\left(\delta_{g} \circ C_{\left(G, A_{G}\right)} \times \mathrm{id}\right)=\psi \circ\left(\mathscr{E}_{g} \times \mathrm{id}\right)
$$

and whose underlying continuous map is $\tilde{\psi}_{g}: q \mapsto \tilde{\psi}(g, q)$. By the methods of $\S 2$, one proves that

$$
\begin{array}{cc}
\left(\psi_{g}\right)^{-1}=\psi_{g^{-1}}, & \psi_{g} \circ \psi_{h}=\psi_{g h}, \\
\psi_{p} \circ R_{g}=\psi_{\tilde{\psi}(g, p)}, & \psi_{g} \circ \psi_{p}=\psi_{p} \circ L_{g} .
\end{array}
$$

4.2. Definition. Let $\left(G, A_{G}\right)$ be a Lie supergroup acting on the supermanifold $\left(M, A_{M}\right)$ via $\psi$ as in(1). We shall say that the action is transitive if there exists a point $p \in M$ such that $\psi_{p}:\left(G, A_{G}\right) \rightarrow\left(M, A_{M}\right)$ is an epimorphism (that is, if for any supermanifold $\left(N, A_{N}\right)$ and for any pair of supermanifold morphisms $\left.\alpha, \beta:\left(M, A_{M}\right) \rightarrow\left(N, A_{N}\right), \alpha \circ \psi_{p}=\beta \circ \psi_{p} \Rightarrow \alpha=\beta\right)$.

Note that if $\psi_{p}$ is an epimorphism for some $p \in M$, then $\psi_{q}$ is an epimorphism for any $q \in M$. Indeed, $\psi$ transitive implies $\tilde{\psi}$ transitive and hence, $\exists g \in G$ such that $q=\tilde{\psi}(g, p)$; since $R_{g}$ is a superdiffeomorphism, $\psi_{q}=\psi_{p} \circ R_{g}$ is an epimorphism.

4.3. Observation. Roughly speaking, one would like to define the orbit through $p$ as the image of the morphism $\psi_{p}$ and the isotropy subsupergroup at $p$ as the locus in $\left(G, A_{G}\right)$ on which the morphisms $\psi_{p}$ and $\mathscr{E}_{p}$ coincide. The definitions, however, have to be given through some careful sheaf-theoretic arguments; the fundamental reason is that in supermanifold theory the values of a given morphism on all the points of the underlying domain do not determine the morphism completely, as has been stressed in $[2-4,10]$. What does determine it is a knowledge of the superalgebra map from the global sections of the sheaf of the target into the global sections of the direct image sheaf of the source. Taking this fact into account, it is reasonable to expect that if we are given an action of $\left(G, A_{G}\right)$ on $\left(M, A_{M}\right)$ as in (1), we should be able to extract a supermanifold (and, in fact, a supergroup) structure on the underlying isotropy subgroup $G_{p}=\left\{g \in G: \tilde{\psi}_{p}(g)=p\right\}$ from the condition

$$
\operatorname{Image}\left(\pi_{p}^{\#}\right)=\operatorname{Image}\left(\mathscr{E}_{p}^{\#}\right) \text {, }
$$

where $\psi_{p}^{\#}$ and $\mathscr{E}_{p}^{\#}$ are the superalgebra maps determined from their corresponding sheaf morphisms. (At this point, the reader might want to look first at the explicit examples given in $\S 5$ below and come back for the general argument later.) 
Once the problem is put this way, the superalgebra of global sections of the isotropy subsupergroup is, if it exists at all, a coequalizer for the diagram

$$
A_{M}(M) \underset{\mathscr{C}_{p}^{\#}}{\stackrel{\psi_{p}^{\#}}{\rightrightarrows}} A_{G}(G) \text {. }
$$

Hence, up to isomorphism, it must be the superalgebra

$$
\left(\tilde{\psi}_{p^{*}} A_{G}\right)(M) / \operatorname{Im}\left(\psi_{p}^{\#}-\mathscr{E}_{p}^{\#}\right)(M)
$$

where

$$
\operatorname{Im}\left(\psi_{p}^{\#}-\mathscr{E}_{p}^{\#}\right)(M)=\left\{\psi_{p}^{\#}(f)-\mathscr{E}_{p}^{\#}(f): f \in A_{M}(M)\right\}
$$

and, of course,

$$
\left(\tilde{\psi}_{p^{*}} A_{G}\right)(M)=A_{G}\left(\tilde{\psi}_{p}^{-1}(M)\right)=A_{G}(G) .
$$

4.4. Observation. What is implied here is that the isotropy subsupergroup of the given action must be defined as an object, $\left(G_{p}, A_{G_{p}}\right)$, together with a morphism

$$
i_{p}:\left(G_{p}, A_{G_{p}}\right) \rightarrow\left(G, A_{G}\right)
$$

that makes the diagram

$$
\begin{array}{cc}
\left(G_{p}, A_{G_{p}}\right) \stackrel{\text { unique }}{\longrightarrow} & (\{*\}, \mathbf{R}) \\
i_{p} \downarrow & \downarrow \delta_{p} \\
\left(G, A_{G}\right) \stackrel{\psi_{p}}{\longrightarrow}\left(M, A_{M}\right)
\end{array}
$$

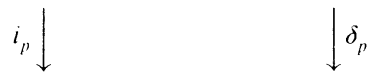

commute, and having the following universal property: for any other object $\left(H, A_{H}\right)$ and morphism $j:\left(H, A_{H}\right) \rightarrow\left(G, A_{G}\right)$ making commutative an analogous square, the existence of a unique morphism

$$
\mu:\left(H, A_{H}\right) \rightarrow\left(G_{p}, A_{G_{p}}\right)
$$

can be deduced, with $i_{p} \circ \mu=j$ (in particular, the morphism $i_{p}$ is necessarily monic). Therefore, the problem of determining both the superalgebra of global sections $A_{G_{p}}\left(G_{p}\right)$ and the morphism $i_{p}$ is that of constructing a pushout diagram for

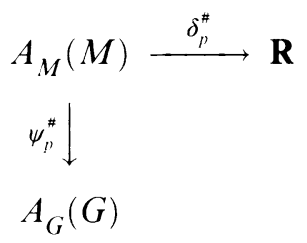

Or, since $\mathscr{E}_{p}^{\#}$ is the composition $A_{M}(M) \rightarrow \mathbf{R} \rightarrow A_{G}(G)$ of $\delta_{p}^{\#}$ followed by the unique map $\mathbf{R} \rightarrow A_{G}(G)$, the problem is that of constructing a coequalizer for diagram (8). Our immediate goal is to prove in the next few paragraphs that, indeed, (9) carries all the information needed to define an embedded subsupergroup of $\left(G, A_{G}\right)$. The first observation to be made is the following: 
4.5. Proposition. There is a natural bijection

$$
\begin{aligned}
G_{p} & =\left\{g \in G: \check{\psi}_{p}(g)=p\right\} \\
& \leftrightarrow\left\{\text { Superalgebra maps }\left(\tilde{\psi}_{p^{*}} A_{G}\right)(M) / \operatorname{Im}\left(\psi_{p}^{\#}-\mathscr{E}_{p}^{\#}\right)(M) \rightarrow \mathbf{R}\right\} .
\end{aligned}
$$

Hence, if the superalgebra of global sections of the sheaf of a supergroup is to be given by (9), its underlying smooth manifold is, up to diffeomorphism, $G_{p}$ (cf. $[3,4])$.

Proof. Each superalgebra map $\left(\tilde{\psi}_{p^{*}} A_{G}\right)(M) / \operatorname{Im}\left(\psi_{p}^{\#}-\mathscr{E}_{p}^{\#}\right) \rightarrow \mathbf{R}$ corresponds naturaily to a superalgebra map $\left(\tilde{\psi}_{p^{*}} A_{G}\right)(M) \rightarrow \mathbf{R}$ whose kernel contains $\operatorname{Im}\left(\psi_{p}^{\#}-\mathscr{E}_{p}^{\#}\right)$. Since the points of $G$ are in natural one-to-one correspondence with the superalgebra morphisms $A_{G}(G) \rightarrow \mathbf{R}$, via $g \leftrightarrow \delta_{g}^{\#}$, it follows that $\delta_{g}^{\#}$ defines a superalgebra map $\left(\tilde{\psi}_{p^{*}} A_{G}\right)(M) / \operatorname{Im}\left(\psi_{p}^{\#}-\mathscr{E}_{p}^{\#}\right) \rightarrow \mathbf{R}$ if and only if $\operatorname{Ker} \delta_{g}^{\#} \supset \operatorname{Im}\left(\psi_{p}^{\#}-\mathscr{E}_{p}^{\#}\right)$, that is, if and only if

$$
\left(\forall f \in A_{M}(M)\right) \quad \delta_{g}^{\#}\left(\psi_{p}^{\#} f-\mathscr{E}_{p}^{\#} f\right)=\delta_{g}^{\#}\left(\psi_{p}^{\#} f\right)-\delta_{g}^{\#}\left(\mathscr{E}_{p}^{\#} f\right)=0 .
$$

Hence, if and only if $\left(\forall \tilde{f} \in C^{\infty}(M)\right) \quad \tilde{f}\left(\tilde{\psi}_{p}(g)\right)=\tilde{f}\left(\tilde{\mathscr{E}}_{p}(g)\right)$, which obviously holds true, if and only if $\tilde{\psi}_{p}(g)=p=\widetilde{\mathscr{E}}_{p}(g)$, that is, if and only if $g \in G_{p}$.

4.6. Theorem. Let $\tilde{i}_{p}: G_{p} \rightarrow G$ be the embedding of the closed Lie subgroup, $G_{p}$ into $G$. Let $A_{G_{p}}$ be the pullback to $G_{p}$, via $\tilde{i}_{p}$, of the sheafification of the presheaf of superalgebras over $G$,

$$
U \mapsto A_{G}(U) /\left(\tilde{\psi}_{p}^{*} \operatorname{Im}\left(\psi_{p}^{\#}-\mathscr{E}_{p}^{\#}\right)\right)(U),
$$

where $\tilde{\psi}_{p}^{*} \operatorname{Im}\left(\psi_{p}^{\#}-\mathscr{E}_{p}^{\#}\right)$ denotes the pullback to $G$ of the sheaf $\operatorname{Im}\left(\psi_{p}^{\#}-\mathscr{C}_{p}^{\#}\right)$ under $\tilde{\psi}_{p}: G \rightarrow M$. Then $\left(G_{p}, A_{G_{p}}\right)$ is an embedded Lie subsupergroup of $\left(G, A_{G}\right)$. The embedding $i_{p}:\left(G_{p}, A_{G_{p}}\right) \rightarrow\left(G, A_{G}\right)$ is defined by the natural sheaf morphism obtained from the composition

$$
A_{G} \rightarrow A_{G} / \tilde{\psi}_{p}^{*} \operatorname{Im}\left(\psi_{p}^{\#}-\mathscr{E}_{p}^{\#}\right) \rightarrow \tilde{i}_{p^{*}} \tilde{i}_{p}^{*}\left\{A_{G} / \tilde{\psi}_{p}^{*} \operatorname{Im}\left(\psi_{p}^{\#}-\mathscr{E}_{p}^{\#}\right)\right\} .
$$

The supergroup structure is inherited from $\left(G_{p}, A_{G_{p}}\right)$ by defining the composition morphism $\nu:\left(G_{p}, A_{G_{p}}\right) \times\left(G_{p}, A_{G_{p}}\right) \rightarrow\left(G_{p}, A_{G_{p}}\right)$ by way of the diagram

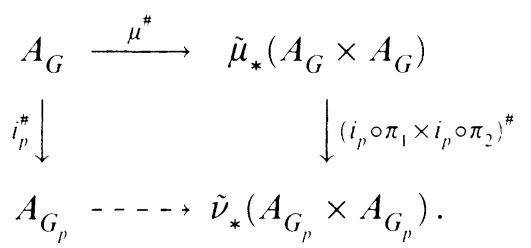

Furthermore, the embedding $i_{p}:\left(G_{p}, A_{G_{p}}\right) \rightarrow\left(G, A_{G}\right)$ has the universal property stated in Observation 4.4 with respect to any other morphism $j:\left(H, A_{H}\right) \rightarrow$ $\left(G, A_{G}\right)$, such that

$$
\psi_{p} \circ j=\delta_{p} \circ C_{\left(H, A_{H}\right)} .
$$


Proof. We shall keep $p \in M$ fixed. The map $\tilde{\psi}_{p}: G \rightarrow M$ can be thought of as a map into the orbit through $p$, say $O_{p}$. We shall endow $O_{p}$ with the topology and differentiable structures that make the induced map $G / G_{p} \rightarrow O_{p}$ a diffeomorphism. In particular, $\tilde{\psi}_{p}$ becomes an open map onto $O_{p}$. This condition is sufficient for the natural $\tilde{\psi}_{p}$-morphism, $A_{G} \rightarrow \tilde{\psi}_{p^{*}} A_{G} \mid O_{p}$ (cf. [16]), to induce isomorphisms on each stalk. In fact, given $g \in G$ and letting $W$ run through all open neighborhoods of $\tilde{\psi}_{p}(g)$, the stalk $\left(A_{G}\right)_{g}$ becomes a target for the direct system defined by the sheaf $\tilde{\psi}_{p^{*}} A_{G}$. Hence, we have the following commutative diagram:

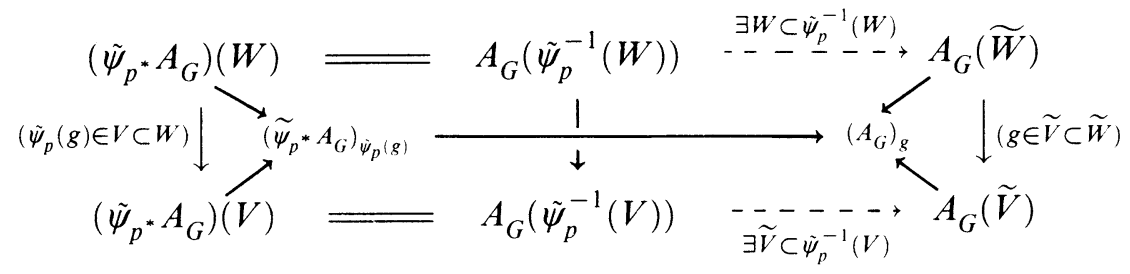

where the dashed horizontal arrows can be drawn simply from the continuity of $\tilde{\psi}_{p}$. But we can reverse the arrow $\left(\tilde{\psi}_{p^{*}} A_{G}\right)_{\tilde{\psi}_{p}(g)} \rightarrow\left(A_{G}\right)_{g}$. Indeed, since $\tilde{\psi}_{p}$ is open, for each open neighborhood $\widetilde{V}$ of $g \in G$ there exists an open neighborhood $V$ of $\widetilde{\psi}_{p}(g) \in M$ with $\widetilde{\psi}_{p}^{-1}(V) \subset \widetilde{V}$; hence, the dashed arrows may be reversed by means of restriction maps. Therefore, $\left(\tilde{\psi}_{p^{*}} A_{G}\right)_{\tilde{\psi}_{p}(g)}$ is a target for the directed system of $A_{G}$. By uniqueness of direct limits, we get

$$
(\forall g \in G) \quad\left(\tilde{\psi}_{p^{*}} A_{G}\right)_{\tilde{\psi}_{p}(g)} \simeq\left(A_{G}\right)_{g} .
$$

Since $\left\{\operatorname{Im}\left(\psi_{p}^{\#}-\mathscr{E}_{p}^{\#}\right)\right\} \tilde{\psi}_{p}(g) \simeq\left\{\tilde{\psi}_{p}^{*} \operatorname{Im}\left(\psi_{p}^{\#}-\mathscr{E}_{p}^{\#}\right)\right\}_{g}$, we furthermore have

$$
\left(\tilde{\psi}_{p^{*}} A_{G}\right)_{\tilde{\psi}_{p}(g)} /\left\{\operatorname{Im}\left(\psi_{p}^{\#}-\mathscr{E}_{p}^{\#}\right)\right\}_{\tilde{\psi}_{p}(g)} \simeq\left(A_{G}\right)_{g} /\left\{\tilde{\psi}_{p}^{*} \operatorname{Im}\left(\psi_{p}^{\#}-\mathscr{E}_{p}^{\#}\right)\right\}_{g}
$$

for all $g \in G$. In particular, we obtain a sheaf epimorphism

$$
A_{G} \rightarrow A_{G} / \tilde{\psi}_{p}^{*} \operatorname{Im}\left(\psi_{p}^{\#}-\mathscr{E}_{p}^{\#}\right) .
$$

Note next that the natural morphism

$$
A_{G} / \tilde{\psi}_{p}^{*} \operatorname{Im}\left(\psi_{p}^{\#}-\mathscr{E}_{p}^{\#}\right) \rightarrow \tilde{i}_{p} \tilde{i}_{p}^{*}\left\{A_{G} / \tilde{\psi}_{p}^{*} \operatorname{Im}\left(\psi_{p}^{\#}-\mathscr{E}_{p}^{\#}\right)\right\}
$$

is an isomorphism. In fact, since $G_{p}$ is a closed Lie subgroup of $G$, the sheaf on the right is the extension of $A_{G_{p}}=\tilde{i}_{p}^{*}\left\{A_{G} / \tilde{\psi}_{p}^{*} \operatorname{Im}\left(\psi_{p}^{\#}-\mathscr{E}_{p}^{\#}\right)\right\}$ by zero (cf. [16]). Therefore, for any open subset $U \subset G$, the map

$$
\begin{gathered}
\tilde{i}_{p^{*}} \tilde{i}_{p}^{*}\left\{A_{G} / \tilde{\psi}_{p}^{*} \operatorname{Im}\left(\psi_{p}^{\#}-\mathscr{E}_{p}^{\#}\right)\right\}(U) \rightarrow \tilde{i}_{p}^{*}\left\{A_{G} / \tilde{\psi}_{p}^{*} \operatorname{Im}\left(\psi_{p}^{\#}-\mathscr{E}_{p}^{\#}\right)\right\}\left(U \cap G_{p}\right), \\
z \mapsto z \mid U \cap G_{p},
\end{gathered}
$$

is an isomorphism and the same conclusion holds true stalkwise. In summary, we get a sheaf epimorphism.

$$
i_{p^{\#}}: A_{G} \rightarrow \tilde{i}_{p^{*}} \tilde{i}_{p}^{*}\left\{A_{G} / \tilde{\psi}_{p}^{*} \operatorname{Im}\left(\psi_{p}^{\#}-\mathscr{E}_{p}^{\#}\right)\right\} .
$$


Now, if this sheaf morphism is to be combined with $\tilde{i}_{p}$ to define a supermanifold morphism, the following compatibility condition must be established:

$$
\left(\forall f \in A_{G}(U)\right)\left(\forall g \in G_{p} \cap U\right) \quad\left(i_{p}^{\#} f\right)^{\sim}(g)=\tilde{f} \circ \tilde{i}_{p}(g) .
$$

This follows easily: by definition of the pullback sheaf, and taking the isomorphism (17) into account, the sections of $\operatorname{Im} i_{p}^{\#}$ over $\operatorname{Im} \tilde{i}_{p} \subset G$ are precisely of the form $\tilde{i}_{p}(g) \mapsto$ germ at $\tilde{i}_{p}(g)$ of some section of the sheaf

$$
A_{G} / \tilde{\psi}_{p}^{*} \operatorname{Im}\left(\psi_{p}^{\#}-\mathscr{E}_{p}^{\#}\right) \text {. }
$$

Since both $A_{G} \rightarrow A_{G} / \tilde{\psi}_{p}^{*} \operatorname{Im}\left(\psi_{p}^{\#}-\mathscr{E}_{p}^{\#}\right)$ and $A_{G} \rightarrow C_{G}^{\infty} \mid G_{p}$ are morphisms of sheaves of superalgebras, the compatibility condition follows.

Now, to show that the supergroup structure $\left\{\mu, \sigma, \mathscr{E}_{e}\right\}$ of $\left(G, A_{G}\right)$ restricts to $\left(G_{p}, A_{G_{p}}\right)$ in the appropriate manner to yield a subsupergroup, we shall make use of the fact that $\mu^{\#}$ is a monomorphism (cf. Lemma 1.9) in combination with the epimorphism $i_{p}^{\#}$. The idea is to show that there is an unambiguous way of closing the following diagram to make it commutative for each pair $(g, h) \in G_{p} \times G_{p}$ :

$$
\begin{aligned}
& \left(A_{G}\right)_{\tilde{\mu}\left(\tilde{i}_{p}(g), \tilde{i}_{p}(h)\right)} \stackrel{\left(\mu^{*}\right)_{\tilde{\mu}_{i}\left(i_{p}(g), i_{p}(h)\right)}^{\longrightarrow}}{\longrightarrow}\left(A_{G} \times A_{G}\right)_{\left(\tilde{i}_{p}(g), \tilde{i}_{p}(h)\right)} \\
& \left(i_{p}^{*}\right)_{\dot{\mu}\left(i_{p}(g) \cdot i_{p}(h)\right)}=\left(i_{p}^{*}\right)_{i_{p}(\dot{\nu}(g . h))} \downarrow \\
& \downarrow\left(i_{p} \circ \pi_{1} \times i_{p} \circ \pi_{2}\right)_{\left(i_{p}(g), i_{p}(h)\right)}^{*} \\
& \left(A_{G_{p}}\right)_{\hat{\nu}(g, h)} \underset{\left(\nu^{*}\right)_{\dot{\nu}(g, h)}}{-} \rightarrow \quad\left(A_{G_{p}} \times A_{G_{p}}\right)_{(g, h)}
\end{aligned}
$$

Note that, since $G_{p}$ is already a Lie subgroup of $G$, its smooth composition map, $\tilde{\nu}: G_{p} \times G_{p} \rightarrow G_{p}$, satisfies $\left(\forall(g, h) \in G_{p} \times G_{p}\right) \tilde{\mu}\left(\tilde{i}_{p}(g), \tilde{i}_{p}(h)\right)=$ $\tilde{i}_{p}(\tilde{\nu}(g, h))$.

Thus, we shall define $\left(\nu^{\#}\right)_{\bar{\nu}(g, h)}$ germwise by going first to $\left(A_{G}\right)_{\tilde{\mu}\left(i_{p}(g), \tilde{i}_{p}(h)\right)}$ using the surjectivity of $\left(i_{p^{*}}\right)_{i_{p}(\hat{\nu}(g, h))}$ and then to $\left(A_{G_{p}} \times A_{G_{p}}\right)_{(g, h)}$ by just following the arrows in the diagram. The only point that has to be checked is that this prescription is well defined; that is,

(20) $\xi \in \operatorname{Ker}\left(i_{p}^{\#}\right)_{\tilde{\mu}\left(\tilde{i}_{p}(g), \hat{i}_{p}(h)\right)} \Rightarrow\left(i_{p} \circ \pi_{1} \times i_{p} \circ \pi_{2}\right)_{\left(i_{p}(g), \tilde{i}_{p}(h)\right)}^{\#}\left(\mu^{\#}\right)_{\tilde{\mu}\left(\tilde{i}_{p}(g), \tilde{i}_{p}(h)\right)} \xi=0$,

which amounts to checking that, for any germ $f_{p} \in\left(A_{M}\right)_{p}$,

$$
\left\{\psi_{p} \circ \mu \circ\left(i_{p} \circ \pi_{1} \times i_{p} \circ \pi_{2}\right)\right\}_{p}^{\#} f_{p}=\left\{\mathscr{E}_{p} \circ \mu \circ\left(i_{p} \circ \pi_{1} \times i_{p} \circ \pi_{2}\right)\right\}_{p}^{\#} f_{p} .
$$

This is so because the morphism $i_{p}^{\#}$ has been defined to have its kernel isomorphism to $\operatorname{Im}\left(\psi_{p}^{\#}-\mathscr{E}_{p}^{\#}\right)$. In fact, since (17) is an isomorphism, we have

$$
\begin{aligned}
\operatorname{Ker}\left(i_{p}^{\#}\right)_{\tilde{\mu}\left(i_{p}(g), i_{p}(h)\right)} & \simeq\left(\tilde{\psi}_{p}^{*} \operatorname{Im}\left(\psi_{p}^{\#}-\mathscr{E}_{p}^{\#}\right)\right)_{\tilde{\mu}\left(i_{p}(g), i_{p}(h)\right)} \\
& \simeq\left(\operatorname{Im}\left(\psi_{p}^{\#}-\mathscr{E}_{p}^{\#}\right)\right)_{\tilde{\psi}\left(\tilde{\mu}\left(i_{p}(g), i_{p}(h)\right)\right)} \\
& \simeq\left(\operatorname{Im}\left(\psi_{p}^{\#}-\mathscr{E}_{p}^{\#}\right)\right)_{p} .
\end{aligned}
$$


More generally, $\operatorname{Ker}\left(i_{p}^{\#}\right)_{g} \simeq\left(\operatorname{Im}\left(\psi_{p}^{\#}-\mathscr{E}_{p}^{\#}\right)\right)_{\dot{\psi}_{p}(g)}$ holds true for any $g \in G$. In particular,

$$
\left(\psi_{p} \circ i_{p}\right)^{\#} f=i_{p}^{\#}\left(\psi_{p}^{\#} f\right)=i_{p}^{\#}\left(\psi_{p}^{\#} f-\mathscr{E}_{p}^{\#} f+\mathscr{E}_{p}^{\#} f\right)=i_{p}^{\#}\left(\mathscr{E}_{p}^{\#} f\right)=\left(\mathscr{E}_{p} \circ i_{p}\right)^{\#} f
$$

for each $f \in A_{M}(M)$, and therefore

$$
\psi_{p} \circ i_{p}=\mathscr{E}_{p} \circ i_{p} .
$$

But now (21) follows when this identity is used in conjunction with the results of the following:

4.7. Lemma. Let $\left(G, A_{G}\right)$ be a Lie supergroup acting on the supermanifold $\left(M, A_{M}\right)$ via the morphism $\psi$ as in (1), and let $p \in M$ be a given point. Let $\left\{\mu, \sigma, \mathscr{E}_{e}\right\}$ be the multiplication, inversion, and identity morphisms of $\left(G, A_{G}\right)$. Let $q_{1}$ and $q_{2}$ be the projection morphisms of the product $\left(G, A_{G}\right) \times\left(G, A_{G}\right)$ onto the first and second factors, respectively. Then

(i) $\mathscr{E}_{p} \circ \mu=\mathscr{E}_{p} \circ q_{1}=\mathscr{E}_{p} \circ q_{2}$,

(ii) $\psi_{p} \circ \mu=\psi \circ\left(q_{1} \times \psi_{p} \circ q_{2}\right)$,

(iii) $\psi \circ\left(\sigma \times \psi_{p}\right)=\psi \circ\left(\mathscr{E}_{e} \times \mathscr{E}_{p}\right)=\mathscr{E}_{p}$.

Proof. The proof of (i) reduces to computing the effect of $\left(\mathscr{E}_{p} \circ \mu\right)^{\#},\left(\mathscr{E}_{p} \circ q_{1}\right)^{\#}$, and $\left(\mathscr{E}_{p} \circ q_{2}\right)^{\#}$ on global sections, but this is simple with the help of (4). Now, for (ii), let $\tau_{i}$ be the projection morphism of the product $\left(G, A_{G}\right) \times\left(G, A_{G}\right) \times$ $\left(G, A_{G}\right)$ onto the $i$ th factor $(i=1,2,3)$. Then

$$
\begin{aligned}
\psi_{p} \circ \mu & =\psi \circ\left(\mathrm{id} \times \mathscr{E}_{p}\right) \circ \mu=\psi \circ\left(\mu \times \mathscr{E}_{p} \circ \mu\right)=\psi \circ\left(\mu \circ\left(q_{1} \times q_{2}\right) \times \mathscr{E}_{p} \circ q_{2}\right) \\
& =\psi \circ\left(\mu \circ\left(\tau_{1} \times \tau_{2}\right) \times \tau_{3}\right) \circ\left(\left(q_{1} \times q_{2}\right) \times \mathscr{E}_{p} \circ q_{2}\right) \\
& =\psi \circ\left(\tau_{1} \times \psi \circ\left(\tau_{2} \times \tau_{3}\right)\right) \circ\left(\left(q_{1} \times q_{2}\right) \times \mathscr{E}_{p} \circ q_{2}\right) \\
& =\psi \circ\left(q_{1} \times \psi \circ\left(q_{2} \times \mathscr{E}_{p} \circ q_{2}\right)\right)=\psi \circ\left(q_{1} \times \psi \circ\left(\mathrm{id} \times \mathscr{E}_{p}\right) \circ q_{2}\right) \\
& =\psi \circ\left(q_{1} \times \psi_{p} \circ q_{2}\right) .
\end{aligned}
$$

Finally, (iii) is proved similarly; one only has to note that $\mathscr{E}_{p} \circ \sigma=\mathscr{E}_{p}$.

We shall now conclude the proof of Theorem 4.6 with a result of Leites that shows that a coordinate neighborhood of the identity can be found in $\left(G, A_{G}\right)$, say $\left(U,\left\{z^{i} ; \eta^{\mu}\right\}\right)$, for which $\left(G_{p} \cap U, A_{G_{p}} \mid G_{p} \cap U\right)$ exhibits the subsupermanifold property. Note that just as in the smooth theory, it is enough to restrict ourselves to such a neighborhood, since we can propagate this local supermanifold structure to the entire embedded subgroup $G_{p}$ via left translations. The result that we invoke is the following:

4.8. Lemma (Leites). Let $U \subset G$ be some open coordinate neighborhood of $e \in M$ and let $\left.I_{p}\right|_{U}$ be the restriction to $U$ of the sheaf of ideals $\tilde{\psi}_{p}^{*} \operatorname{Im}\left(\psi_{p}^{\#}-\mathscr{E}_{p}^{\#}\right)$ in $A_{G}$. Let

$$
H_{p}=\left\{g \in U: \forall f \in I_{p}(U), \tilde{f}(g)=0\right\}
$$


For each $g \in H_{p}$, there is an open neighborhood $V(U \supset V \in g)$ and there are homogeneous sections $\left\{f^{1}, \ldots, f^{k} ; \eta^{1}, \ldots, \eta^{l}\right\}, f^{j} \in\left(I_{p}(V)\right)_{0}$, and $\eta^{\nu} \in$ $\left(I_{p}(V)\right)_{1}$ that generate $I_{p}(V)$. Furthermore, $\left\{\left(d f^{1}\right)_{g}, \ldots,\left(d f^{k}\right)_{g} ;\left(d \eta^{1}\right)_{g}, \ldots\right.$, $\left.\left(d \eta^{l}\right)_{g}\right\}$ is a linearly independent set and therefore $\left\{f^{1}, \ldots, f^{k} ; \eta^{1}, \ldots, \eta^{l}\right\}$ may be extended to a coordinate system $\left\{f^{1}, \ldots, f^{k}, f^{k+1}, \ldots, f^{m} ; \eta^{1}, \ldots\right.$, $\left.\eta^{l}, \eta^{l+1}, \ldots, \eta^{n}\right\}$ on $V$.

Proof (cf. [4]). Note that because of Proposition 4.5, $H_{p}$ above is precisely $G_{p} \cap U$. Therefore, noting that the assertion regarding the universal character of $\left(G_{p}, A_{G_{p}}\right)$ is automatic by the very definition of $A_{G_{p}}$ (cf. Observations 4.3 and 4.4), Theorem 4.6 follows.

4.9. Observation. Let us briefly discuss how the orbits of an action $\psi$ are to be understood. Following [3], the idea is to show that a natural supermanifold sheaf can be defined on the space of cosets $G / G_{p}$ and then carry this structure over the orbit $O_{p}=\tilde{\psi}_{p}(G) \subset M$, via $\tilde{\psi}_{p}$, so as to have $\left(G / G_{p}, A_{G / G_{p}}\right) \simeq$ $\left(O_{p}, A_{O_{p}}\right)$.

In this way, it suffices to show that for any supergroup $\left(G, A_{G}\right)$ and a given embedding of supergroups $i:\left(H, A_{H}\right) \rightarrow\left(G, A_{G}\right)$ (cf. Remark 1.10), there is a natural way of defining a supermanifold sheaf over the coset space $G / H$, say $A_{G / H}$, and a sheaf monomorphism $q^{*}: A_{G / H} \rightarrow \tilde{q}_{*} A_{G}$, where $\tilde{q}: G \rightarrow G / H$ is the canonical projection.

This is obtained as follows (compare with [3]): For any open subset $U \subset$ $G / H$, the assignment

$$
\begin{aligned}
U & \mapsto\left\{f \in A_{G}\left(\tilde{q}^{-1}(U)\right) \mid(\forall h \in H) R_{h}^{\#} f=f\right\} \\
& =\bigcap_{h \in H} \operatorname{Ker}\left(R_{h}^{\#}-\mathrm{id}^{\#}\right)\left(\tilde{q}^{-1}(U)\right)
\end{aligned}
$$

defines a sheaf of superalgebras over $G / H$. It is, in fact, a subsheaf of $\tilde{q}_{*} A_{G}$. This is precisely the sheaf $A_{G / H}$ and $q^{\#}$ is simply the natural inclusion into $\tilde{q}_{*} A_{G}$. Note, for example, that if $i:\left(H, A_{H}\right) \rightarrow\left(G, A_{G}\right)$ is the embedding of the isotropy subsupergroup of a transitive action $\psi$ as in (1) (cf. Definition $4.2)$, then the sheaf defined in $(24)$ is isomorphic to $\operatorname{Im} \psi_{p}^{\#}$, in view of the third relation in (6).

\section{EXAMPLES}

5.1. Example. $\mathrm{GL}_{s}(1 \mid 1)$ acting on the supermanifold $\mathbf{R}^{2 / 2}=\left(\mathbf{R}^{2}, R^{2 \mid 2}\right)$ (cf [12]). Let

$$
\psi:\left(\mathrm{GL}(2), \mathrm{GL}_{s}(1 \mid 1)\right) \times\left(\mathbf{R}^{2}, R^{2 \mid 2}\right) \rightarrow\left(\mathbf{R}^{2}, R^{2 \mid 2}\right)
$$

be the action in Example 3.2. To simplify the writing, we shall redefine the 
coordinates introduced in Example 1.4 as follows:

$$
\begin{aligned}
& A=a, \quad \pi \Gamma=b ; \quad \pi A=\alpha, \quad \Gamma=\beta ; \\
& \pi \Theta=c, \quad D=d ; \quad \Theta=\gamma, \quad \pi D=\delta .
\end{aligned}
$$

Similarly, if $\{x, \pi \xi\}$ and $\{\pi x, \xi\}$ are respectively the even and odd (linear) coordinates in $\mathbf{R}^{2 \mid 2}$ introduced in 3.2, we redefine $\pi \xi$ and $\pi x$ and write instead

$$
\pi \xi=y, \quad \pi x=\zeta .
$$

The action morphism of 3.2 is then given by

$$
\psi^{\#}\left(\begin{array}{l}
x+\zeta \\
y+\xi
\end{array}\right)=\left(\begin{array}{ll}
a+\alpha & b+\beta \\
c+y & d+\delta
\end{array}\right)\left(\begin{array}{l}
x+\zeta \\
y+\xi
\end{array}\right)
$$

where we have further omitted the explicit reference to the projection morphisms of the product supermanifold $\mathrm{GL}_{s}(1 \mid 1) \times \mathbf{R}^{2 \mid 2}$. If we now fix some $p \in \mathbf{R}^{2}$ and consider

$$
\psi_{p}=\psi \circ\left(\mathrm{id} \times \delta_{p} \circ c\right): \mathrm{GL}_{s}(1 \mid 1) \rightarrow \mathbf{R}^{2 \mid 2}
$$

we find that

$$
\begin{array}{ll}
\psi_{p}^{\#} x=a \tilde{x}(p)+b \tilde{y}(p), & \psi_{p}^{\#} \zeta=\alpha \tilde{x}(p)+\beta \tilde{y}(p), \\
\psi_{p}^{\#} y=c \tilde{x}(p)+d \tilde{y}(p), & \psi_{p}^{\#} \xi=\gamma \tilde{x}(p)+\delta \tilde{y}(p) .
\end{array}
$$

In particular, note that the map $\tilde{\psi}_{p}: \operatorname{GL}(4) \rightarrow \mathbf{R}^{2}$ is given by

$$
\tilde{\psi}_{p}\left(\left(\begin{array}{ll}
a & b \\
c & d
\end{array}\right)\right)=\left(\begin{array}{ll}
a & b \\
c & d
\end{array}\right)\left(\begin{array}{l}
\tilde{x}(p) \\
\tilde{y}(p)
\end{array}\right) .
$$

Now, consider the morphism $\mathscr{E}_{p}: \mathrm{GL}_{s}(1 \mid 1) \rightarrow \mathbf{R}^{2 \mid 2}$. According to 4.1, its effect on the coordinate functions is this:

$$
\begin{array}{ll}
\mathscr{E}_{p}^{\#} x=\tilde{x}(p) 1_{\mathrm{GL}(2 \mid 4)}, & \mathscr{E}_{p}^{\#} \zeta=0, \\
\mathscr{E}_{p}^{\#} y=\tilde{y}(p) 1_{\mathrm{GL}(2 \mid 4)}, & \mathscr{E}_{p}^{\#} \xi=0 .
\end{array}
$$

Therefore, the condition $\operatorname{Im}\left(\psi_{p}^{\#}\right)=\operatorname{Im}\left(\mathscr{E}_{p}^{\#}\right)$ imposes the following relations among the coordinates of $\mathrm{GL}_{s}(1 \mid 1)$ :

$$
\begin{aligned}
& \psi_{p}^{\#} x=a \tilde{x}(p)+b \tilde{y}(p)=\tilde{x}(p) 1_{\mathrm{GL}(2 \mid 4)}=\mathscr{E}_{p}^{\#} x, \\
& \psi_{p}^{\#} y=c \tilde{x}(p)+d \tilde{y}(p)=\tilde{y}(p) 1_{\mathrm{GL}(2 \mid 4)}=\mathscr{E}_{p}^{\#} y, \\
& \psi_{p}^{\#} \zeta=a \tilde{x}(p)+\beta \tilde{y}(p)=0=\mathscr{E}_{p}^{\#} \zeta, \\
& \psi_{p}^{\#} \xi=\gamma \tilde{x}(p)+\delta \tilde{y}(p)=0=\mathscr{E}_{p}^{\#} \xi .
\end{aligned}
$$

For example, under the assumption that $\tilde{x}(p)=1$ and $\tilde{y}(p)=0$ (choice of $p$ ), these equations imply that

$$
a=1_{\mathrm{GL}(1 \mid 1)}, \quad c=0, \quad \alpha=0, \quad \gamma=0 .
$$


These conditions indeed define an embedded subsupergroup of $\mathrm{GL}_{s}(1 \mid 1)$, for the set of all matrices of the form

$$
\left(\begin{array}{cc}
1_{\mathrm{GL}(1 \mid 1)} & b+\beta \\
0 & d+\delta
\end{array}\right)
$$

is closed under the composition morphism $\mu$ defined in Example 1.4. Note that the underlying Lie group of such subsupergroup is the semidirect product of the multiplicative group of the nonzero real numbers ( $d$ being a local coordinate) with the additive group of the reals ( $b$ being the corresponding coordinate). Also note that it has odd dimension equal to 2 .

It is worth mentioning that the calculations in this example are only slightly simpler than those required in the more general case of the Lie supergroup $\mathrm{GL}_{s}(m \mid n)$ acting on the supermanifoldification $V_{s}$ of the $(m, n)$-dimensional supervector space $V=V_{0} \oplus V_{1}$. Indeed, one has only to interpret the definitions in (2) and ( 3 ) as equations between matrices of the appropriate sizes and proceed accordingly. For example, the relations (9) look exactly the same in the general case, where $\tilde{x}(p)$ and $\tilde{y}(p)$ represent the column vectors with the coordinates in $V_{0}$ and $V_{1}$ of the point $p$. The relations that define the isotropy subsupergroup, and hence isotropy subsupergroups themselves, vary depending on the chosen point, as expected.

5.2. Example. $\mathrm{GL}(2 \mid 2)=\mathrm{GL}\left(V_{0} \mid V_{1}\right)$ acting on $G_{1 \mid 1}\left(V^{2 \mid 2}\right), V^{2 \mid 2}=V_{0} \oplus V_{1}$ being a $(2,2)$-dimensional supervector space.

This time we shall relabel the coordinates $\left\{A^{b j}, D^{B J}\right\}$ and $\left\{\Gamma^{b J}, \Theta^{B j}\right\}$ of Example 1.4 as follows:

$$
\begin{array}{llll}
A^{11}=a, & A^{12}=b, & \Gamma^{11}=\alpha, & \Gamma^{12}=\beta, \\
A^{21}=c, & A^{22}=d, & \Gamma^{21}=\gamma, & \Gamma^{22}=\delta, \\
\Theta^{11}=\pi, & \Theta^{12}=\rho, & D^{11}=q, & D^{12}=r, \\
\Theta^{21}=\sigma, & \Theta^{22}=\tau, & D^{21}=s, & D^{22}=t .
\end{array}
$$

Let $\{x, y\}$ and $\{\xi, \zeta\}$, respectively, be the even and odd local coordinates on $G_{1 \mid 1}\left(V^{2 \mid 2}\right)$ defined on the open neighborhood, $\{x \neq 0\} \cap\{y \neq 0\}$. According to Example 3.3 (and omitting the projection morphisms of the product) we have (12)

$$
\begin{aligned}
& \psi^{\#}\left(\begin{array}{ll}
x & \xi \\
\zeta & y
\end{array}\right) \\
& \quad=\left(\left(\begin{array}{ll}
a & \alpha \\
\pi & q
\end{array}\right)\left(\begin{array}{ll}
x & \xi \\
\zeta & y
\end{array}\right)+\left(\begin{array}{ll}
b & \beta \\
\rho & r
\end{array}\right)\right)\left(\left(\begin{array}{ll}
c & \gamma \\
\sigma & s
\end{array}\right)\left(\begin{array}{ll}
x & \xi \\
\zeta & y
\end{array}\right)+\left(\begin{array}{ll}
d & \delta \\
\tau & t
\end{array}\right)\right)^{-1}
\end{aligned}
$$

and the assumption to be made is that we do not leave the same coordinate patch after the transformation. That is, the coordinates are constrained in such a way that $c x+d-\gamma \zeta$ and $s y+t-\sigma \xi$ are both invertible (see [14] for computations). In particular, $c x+d$ and $s y+t$ are assumed to be invertible.

Since we are interested in comparing the image of $\psi_{p}^{\#}$ with that of $\mathscr{E}_{p}^{\#}$, we apply the morphism $\left(\text { id } \times \mathscr{E}_{p}\right)^{\#}$ to both sides of each of (12). After some 
simplification, we find that

$$
\begin{aligned}
& \psi_{p}^{\#} x=\left(\frac{(a \tilde{x}(p)+b)}{(c \tilde{x}(p)+d)}+\frac{(\sigma \tilde{x}(p)+\tau)}{(c \tilde{x}(p)+d)} \frac{(\alpha \tilde{y}(p)+\beta)}{(s \tilde{y}(p)+t)}\right)\left(1-\frac{(\sigma \tilde{x}(p)+\tau)}{(c \tilde{x}(p)+d)} \frac{(\gamma y(p)+\delta)}{(s \tilde{y}(p)+t)}\right), \\
& \psi_{p}^{\#} y=\left(\frac{(q \tilde{y}(p)+r)}{(s \tilde{y}(p)+t)}+\frac{(\pi \tilde{x}(p)+\rho)}{(c \tilde{x}(p)+d)} \frac{(\gamma \tilde{y}(p)+\delta)}{(s \tilde{y}(p)+t)}\right)\left(1-\frac{(\sigma \tilde{x}(p)+\tau)}{(c \tilde{x}(p)+d)} \frac{(\gamma \tilde{y}(p)+\delta)}{(s \tilde{y}(p)+t)}\right), \\
& \psi_{p}^{\#} \xi=\left(\frac{(\alpha \tilde{y}(p)+\beta)}{(s \tilde{y}(p)+t)}-\frac{(a \tilde{x}(p)+b)}{(c \tilde{x}(p)+d)} \frac{(\gamma \tilde{y}(p)+\delta)}{(s \tilde{y}(p)+t)}\right)\left(1-\frac{(\sigma \tilde{x}(p)+\tau)}{(c \tilde{x}(p)+d)} \frac{(\gamma \tilde{y}(p)+\delta)}{(s \tilde{y}(p)+t)}\right), \\
& \psi_{p}^{\#} \zeta=\left(\frac{(\pi \tilde{x}(p)+\rho)}{(c \tilde{x}(p)+d)}-\frac{(\sigma \tilde{x}(p)+\tau)}{(c \tilde{x}(p)+d)} \frac{(p \tilde{y}(p)+r)}{(s \tilde{y}(p)+t)}\right)\left(1-\frac{(\sigma \tilde{x}(p)+\tau)}{\left(c^{\prime}(p)+d\right)} \frac{(\gamma \tilde{y}(p)+\delta)}{(s \tilde{y}(p)+t)}\right) .
\end{aligned}
$$

We now choose the point $p$ as that whose coordinates are

$$
\tilde{x}(p)=0 \quad \text { and } \quad \tilde{y}(p)=0
$$

so that

$$
\mathscr{E}_{p}^{\#} x=\tilde{x}(p)=0, \quad \mathscr{E}_{p}^{\#} y=\tilde{y}(p)=0, \quad \mathscr{E}_{p}^{\#} \xi=0, \quad \mathscr{E}_{p}^{\#} \zeta=0 .
$$

It is then easy to verify that the condition $\operatorname{Im}\left(\psi_{p}^{\#}\right)=\operatorname{Im}\left(\mathscr{E}_{p}^{\#}\right)$ yields the coordinate relations

$$
A^{12}=b=0, \quad \Gamma^{12}=\beta=0, \quad \Theta^{12}=\rho=0, \quad D^{12}=r=0 .
$$

But these conditions define an embedded $(6,6)$-dimensional subsupergroup of the $(8,8)$-dimensional supergroup $\mathrm{GL}(2 \mid 2)$.

We remark again that most of the computations above remain valid (and the final results take exactly the same form) for the more general case of the supergroup $\mathrm{GL}(m \mid n)$ acting on the supergrassmannian $G_{k \mid h}\left(V^{m \mid n}\right)$. All that is required is to interpret $A^{11}, A^{12}, \ldots, D^{21}, D^{22}$ in (11) as block matrices of the appropriate sizes. What comes out of the same analysis is that for the point $p$ whose coordinates are given by (14) (understood as equations between matrices), the condition $\operatorname{Im}\left(\psi_{p}^{\#}\right)=\operatorname{Im}\left(\mathscr{E}_{p}^{\#}\right)$ yields the same coordinate relations (16), to be interpreted as conditions on the corresponding blocks of coordinates in the supergroup. The only expressions that look different in the general case are those in (13), where we have used quotients and have permuted some of the factors, but the reader will have not trouble in finding what the general expressions should be. In fact, he/she will note that the common factor on the right of (13) is invertible. Taking this into account, it is not difficult to see then that (16) defines an embedded subsupergroup of $\mathrm{GL}(m \mid n)$ of dimension

$$
\left(m^{2}+n^{2}-k(m-k)-h(n-h), 2 m n-h(m-k)-k(n-h)\right) .
$$

\section{REFERENCES}

1. M. Batchelor, The structure of supermanifolds, Trans. Amer. Math. Soc. 253 (1980), 329338.

2. F. A. Berezin, Introduction to superanalysis (A. A. Kirillov, ed.), Kluwer Academic Publishers, 1987. 
3. B. Kostant, Graded manifolds, graded Lie theory and prequantization, Lecture Notes in Math., vol. 570, Springer-Verlag, Berlin, 1977, pp. 177-306.

4. D. A. Leites, Introduction to the theory of supermanifolds, Russian Math. Surveys 35 (1980), $1-64$.

5. __ Seminar on supermanifolds, no. 31; Report from the Department of Mathematics, Univ. of Stockholm, Sweden, 1987.

6. Yu. I. Manin, Holomorphic supergeometry and Yang-Mills superfields, preprint.

7. __ Gauge field theory and complex geometry, Nauka, Moscow, 1984; English transl., Springer-Verlag, New York, 1988.

8. __. Grassmannians and flags in supergeometry, Amer. Math. Soc. Transl. (2) 137 (1987), 87-99.

9. ___ Flag superspaces and supersymmetric Yang-Mills equations, Arithmetic and Geometry: Papers Dedicated to I. R. Shafarevich on the Occasion of his 60th Birthday, vol. II (M. Artin and J. Tate, eds.), Birkhäuser, Boston, Mass., 1983.

10. M. Rothstein, The axioms of supermanifolds and a new structure arising from them, Trans. Amer. Math. Soc. 297 (1986), 159-180.

11. O. A. Sanchez Valenzuela, On supergeometric structures, Harvard thesis, May 1986, and On supervector bundles, IIMAS-UNAM preprint 457, Mexico, 1986.

12. __ Linear supergroup actions. I: On the defining properties, Trans. Amer. Math. Soc. $\mathbf{3 0 7}$ (1988), 569-595.

13. __ On Grassmannian supermanifolds, Trans. Amer. Math. Soc. 307 (1988), 597-614.

14. __ Matrix computations in linear superalgebra, Linear Algebra Appl. (to appear).

15. T. Schmitt, Super differential geometry, Akademie der Wissenschaften der DDR, Institut für Mathematik, report R-MATH-05/84, Berlin, 1984.

16. O. Tennison, Sheaf theory, London Math. Soc. Lecture Notes Ser., no. 20, Cambridge Univ. Press, Cambridge, 1975.

Department of Mathematics and Statistics, University of New Mexico, Albuquerque, New MeXico 87131

Centro de Investigación en Matemáticas, A.D., Guanajuato, Gto., México 